\title{
ON THE CONSTRUCTION AND DISTRIBUTION OF A LOCAL MARTINGALE WITH A GIVEN ABSOLUTE VALUE \\ BY
}

EDWIN PERKINS

\begin{abstract}
A local martingale is constructed on an appropriate Loeb space whose absolute value equals a given nonnegative local submartingale. Nonstandard analysis is used to reduce the problem to the discrete time setting where the original construction of D. Gilat is fairly simple. This approach has the advantage of allowing explicit computations. In particular, the distribution of the local martingale is described in terms of the Doob-Meyer decomposition of the original local submartingale.
\end{abstract}

1. Introduction and statement of results. In [6] D. Gilat proved that every nonnegative submartingale $x$ is equal in law to the absolute value of a martingale $m$. His construction, however, did not shed any light on the nature of this martingale. Several other authors, including Protter and Sharpe [13], Maisonneuve [11], and Barlow and Yor [4] have given more transparent constructions of $m$ on an enlargement of the space on which $x$ is defined (by an enlargement of $(\Omega, \mathscr{F}, P)$ we mean a space of the form $\left.\left(\Omega \times \Omega^{\prime}, \mathscr{F} \times \mathscr{F}^{\prime}, P \times P^{\prime}\right)\right)$. These constructions have made additional assumptions on $x$-notably $x(t) \wedge x\left(t^{-}\right)>0$ for all $t \geqslant 0$, which is the case in [11 and 13]. Recently M. T. Barlow has shown in [3] that if $x$ is a nonnegative submartingale on $\left(\Omega, \mathscr{F}, P, \mathscr{F}_{t}\right)$ and if there is a random variable on $(\Omega, \mathscr{F}, P)$ which has a continuous distribution function and is independent of $V_{t \geqslant 0} \mathscr{F}_{t}$, then there is a filtration $\left\{\mathscr{N}_{t} \mid t \geqslant 0\right\}$ and a martingale $m$ on $\left(\Omega, \mathscr{F}, P, \Re_{t}\right)$ such that $|m|=x$, $\mathscr{F}_{t} \subset \mathscr{N}_{t}$, and every $\mathscr{F}_{t}$-martingale is an $\mathscr{N}_{t}$-martingale. It is our aim to use nonstandard analysis to give an intuitive construction of a (local) martingale, $m$, whose absolute value equals a given nonnegative (local) submartingale, $x$. Our construction was carried out independently of Barlow's work and complements his results since the finite-dimensional distribution of $m$ are described in terms of the Doob-Meyer decomposition of $x$. The construction uses nonstandard analysis to reduce the continuous time problem to a discrete time problem, where the construction of Gilat is fairly simple.

To state a precise version of the main result we will need the following definition that appears in Aldous [1]. The notation $\left(\Omega, \mathscr{F}, P, \mathscr{F}_{t}\right)$ indicates that $\left\{\mathscr{F}_{t} \mid t \geqslant 0\right\}$ is a right-continuous filtration on the complete probability space $(\Omega, \mathscr{F}, P)$ and that $\mathscr{F}_{0}$ includes all the $P$-null sets.

Received by the editors August 11, 1980 and, in revised form, March 10, 1981. This work was presented to the American Mathematical Society at its winter meeting in January 1980.

AMS (MOS) subject classifications (1970). Primary 60G45, 02H25.

Key words and phrases. Submartingale, local martingale, nonstandard analysis.

(C)1982 American Mathematical Society 0002-9947/82/0000-1013/\$06.25 
Definition 1.1. Suppose $x^{i}$ is an adapted process on $\left(\Omega^{i}, \mathscr{F}^{i}, P^{i}, \mathscr{F}_{t}^{i}\right)(i=1,2)$ with sample paths in

$$
D(M)=\{y:[0, \infty) \rightarrow M \mid y(\cdot) \text { is right continuous with left limits }\},
$$

where $M$ is a Polish space. If $\phi: D(M) \rightarrow \mathbf{R}^{d}$ is bounded and Borel measurable (give $D(M)$ the Skorokhod $J_{1}$ topology as in Billingsley [5] and Stone [14]), let $L_{\phi}^{i}(t)$ denote a right-continuous version of $E^{i}\left(\phi\left(x^{i}\right) \mid \mathscr{F}_{t}^{i}\right)(i=1,2)$. Then $\left(x^{1}, \mathscr{F}^{1}\right)$ and $\left(x^{2}, \mathscr{F}^{2}\right)$ are synonomous (we write $\left.\left(x^{1}, \mathscr{F}_{.}^{1}\right) \equiv\left(x^{2}, \mathscr{F}_{.}^{2}\right)\right)$ if $\mathcal{L}\left(L_{\phi}^{1}\right)=\mathcal{L}\left(L_{\phi}^{2}\right)$ for all $\phi$ and $d$, where $\mathcal{L}\left(L_{\phi}^{i}\right)$ is the law of $L_{\phi}^{i}$ on $D\left(\mathbf{R}^{d}\right)$.

The above definition is easily shown to be equivalent to that in Aldous [1]. Note that if $\left(x^{1}, \widetilde{F}_{.}^{1}\right) \equiv\left(x^{2}, \mathscr{F}_{.}^{2}\right)$, then $x^{1}$ and $x^{2}$ have the same distribution on $D(M)$.

Our main result, which holds with or without the expressions in parentheses, is the following:

THEOREM 1.2. If $y$ is a nonnegative (local) submartingale on $\left(X, \mathcal{G}, Q, \mathcal{G}_{t}\right)$, there is a (local) martingale $m$ on some $\left(\Omega, \mathscr{F}, P, \mathscr{F}_{t}\right)$ such that

$$
\left((|m|, a), \mathscr{F}_{.}\right) \equiv\left((y, b), \mathcal{G}_{.}\right),
$$

where $a$ and $b$ are the increasing processes in the Doob-Meyer decompositions of $|m|$ and $y$, respectively. Moreover, $m$ may be chosen so that

$$
E(m(0) \| m \mid, a)=0 \text { a.s., }
$$

and if $e_{0} \in\{-1,0,1\},\left\{e_{1}, \ldots, e_{n}\right\} \subset\{-1,1\}, B_{0}, \ldots, B_{n} \in \mathscr{乃}((0, \infty))$, and $0=t_{0}<t_{1}$ $<\cdots<t_{n}$, then

$$
\begin{aligned}
& P\left(\left(m\left(t_{0}\right), \ldots, m\left(t_{n}\right)\right) \in e_{0} B_{0} \times \cdots \times e_{n} B_{n}\right) \\
& \quad=\int I\left(\left(y\left(t_{0}\right), y\left(t_{1}\right), \ldots, y\left(t_{n}\right)\right) \in\left|e_{0}\right| B_{0} \times B_{1} \times \cdots \times B_{n}\right)
\end{aligned}
$$

$$
\begin{aligned}
\times\left(e_{0}^{2}+1\right)^{-1} 2^{-n} \prod_{i=1}^{n}\left(1+e_{i-1} e_{i} \exp \left\{-\int_{t_{i-1}}^{t_{i}} y(s)^{-1} d b^{c}(s)\right\}\right. \\
\left.\times \prod_{t_{i-1}<s \leqslant t_{i}}\left(1-\left(y\left(s^{-}\right)+\Delta b(s)\right)^{-1} \Delta b(s)\right)\right) d Q,
\end{aligned}
$$

where $b^{c}(t)=\int_{0}^{t} I(\Delta b(s)=0) d b(s)$.

Since the filtration $\left\{\mathcal{G}_{t}\right\}$ is an integral part of the submartingale $y$, it seems natural to require that the filtration for $m,\left\{\mathscr{F}_{t}\right\}$, be equivalent to $\left\{\mathcal{G}_{t}\right\}$ insofar as they relate to $(|m|, a)$ and $(y, b)$, respectively. This formulation of the existence problem has the advantage of leading to corresponding uniqueness results which are presented in [12].

In $\S 2$ we construct a local martingale on a Loeb space whose absolute value equals a given nonnegative local submartingale. The finite-dimensional distributions of $m$ are computed in $\S 3$. The proof of Theorem 1.2 then amounts to showing that the class of probability spaces considered in $\S 2$ is large enough. In $\S 4$ we establish an important property of $m$, which in [12] is shown to uniquely determine several 
additional properties of any local martingale $\left(m(t), \mathscr{F}_{t}\right)$ that satisfies $(1.1)$. 55 contains the proof of a technical lifting theorem needed in $\$ 2$.

The presentation assumes a knowledge of nonstandard methods in probability theory. A good introduction to the subject may be found in Loeb [10]. Keisler [8] and Hoover and Perkins [7] contain further background material.

2. Construction of the local martingale. Our setting is that used in Hoover and Perkins [7] (hereafter abbreviated [H.P.]). We work in an $\omega_{1}$-saturated enlargement of a superstructure $V(S)$, where $\mathbf{R} \subset S$. Consider an internal probability space $\left(\Omega^{1}, \mathbb{Q}^{1}, \bar{P}^{1}\right)$ on which $\left\{\mathbb{Q}_{t}^{1} \mid \underline{t} \in T\right\}$ is a nondecreasing internal sequence of ${ }^{*}$-sub- $\sigma$ fields indexed by $T=\left\{k \Delta t \mid k \in{ }^{*} \mathbf{N}_{0}\right\}$ ( $\Delta t$ is a fixed positive infinitesimal and $\left.{ }^{*} \mathbf{N}_{0}={ }^{*} \mathbf{N} \cup\{0\}\right)$. Elements of $T$ are denoted by $\underline{t}, \underline{u}, \underline{v}$, etc. The Loeb space generated by the above space is $\left(\Omega^{1}, \mathscr{F}^{1}=L\left(\mathbb{Q}^{1}\right), P^{1}=L\left(\bar{P}^{1}\right)\right)$ (i.e., $P^{1}$ is the unique extension of ${ }^{\circ} \bar{P}^{1}$ to $\sigma\left(\mathbb{Q}^{1}\right)$, and $\mathscr{F}^{1}$ is the $P^{1}$-completion of $\left.\sigma\left(\mathbb{Q}^{1}\right)\right)$, and a filtration on $\left(\Omega^{1}, \mathscr{F}^{1}\right)$ is defined by

$$
\mathscr{F}_{t}^{1}=\left(\bigcap_{\underline{o}>t} \sigma\left(\mathscr{Q}_{\underline{t}}^{1}\right)\right) \vee \Re^{1},
$$

where $\mathcal{T}^{1}$ is the class of all $P^{1}$-null sets. It is clear that $\left\{\mathscr{F}_{t}^{1} \mid t \geqslant 0\right\}$ is right continuous. The 4-tuple $\left(\Omega^{1}, \mathscr{F}^{1}, P^{1}, \mathscr{F}_{t}^{1}\right)$ constructed in this manner is called the adapted Loeb space generated by $\left(\Omega^{1}, \mathbb{Q}_{1}, \bar{P}^{1}, \mathbb{Q}_{t}^{1}\right)$. The reader is referred to Loeb [9] and Anderson [2] for background material on Loeb spaces. We will need the following elementary results from [H.P.].

Lemma 2.1 [H. P., Lemma 3.3 and Theorem 3.2]. (a) If $Y$ is an internal $S$-integrable random variable on $\left(\Omega^{1}, \mathbb{Q}^{1}, \bar{P}^{1}\right)$ and $\mathscr{D}$ is an internal ${ }^{*}$-sub- $\sigma$-field of $\mathbb{Q}^{1}$, then

$$
{ }^{\circ} \bar{E}^{1}(Y \mid \mathscr{D})=E^{1}\left({ }^{\circ} Y \mid L(\mathscr{D})\right) \text { a.s. }
$$

(b) If $y: \Omega^{1} \rightarrow \mathbf{R}^{d}$ is $\mathscr{F}_{t}^{1}$-measurable for some $t \geqslant 0$, then there is a $t \approx t$ and an internal $\mathbb{Q}_{\underline{t}}^{1}$-measurable random variable $Y: \Omega^{1} \rightarrow{ }^{*} \mathbf{R}^{d}$ such that ${ }^{\circ} Y=y$ a.s.

Unless otherwise indicated, all stochastic processes have sample paths in $D=D(\mathbf{R})$. Lower case letters denote standard processes on the appropriate Loeb space, while upper case letters are used to denote internal processes on some internal probability space.

Let $x^{1}(t)$ be a nonnegative local submartingale on $\left(\Omega^{1}, \mathscr{F}^{1}, P^{1}, \mathscr{F}_{t}^{1}\right)$. That is, there is a sequence of stopping times $\left\{U_{j}\right\}$, increasing to $\infty$ a.s., such that $x^{1}\left(U_{j} \wedge t\right)$ is an $\left\{\mathscr{F}_{t}^{1}\right\}$-submartingale for all $j$. Since $x^{1}$ is locally of class $D$, it has a Doob-Meyer decomposition $x^{1}=n^{1}+a^{1}$ ( $n^{1}$ is a local martingale and $a^{1}$ is an increasing predictable process that equals zero at $t=0$ ). In practice we will construct $x^{1}$ and $\left(\Omega^{1}, \mathbb{Q}^{1}, \bar{P}^{1}, \mathbb{Q}_{t}^{1}\right)$ from the (local) submartingale, $y$, of Theorem 1.2 as follows. Let

$$
\left(\Omega^{1}, \mathbb{Q}^{1}, \bar{P}^{1}, \mathbb{Q}_{t}^{1}\right)=*\left(X, \mathcal{G}, Q, \mathcal{G}_{t}\right) .
$$

Then $x^{1}$ will be the standard part of $* y(\cdot, \omega)$ in the Skorokhod $J_{1}$ topology. Using Lemma 2.1 and some elementary properties of synonymity, one can show that 
$\left(\left(x^{1}, a^{1}\right), \mathscr{F}^{1}\right) \equiv\left((y, b), \mathcal{G}_{.}\right)$. (The details of these arguments are presented at the end of $\$ 3$ with the proof of Theorem 1.2.) Therefore in order to prove Theorem 1.2, we may replace $\left((y, b), \mathcal{G}_{\text {. }}\right)$ with $\left(\left(x^{1}, a^{1}\right), \mathscr{F}^{1}\right)$. We will construct a local martingale whose absolute value is $x^{1}$ in three steps:

1. Lift $n^{1}$ and $a^{1}$ to internal processes on $T \times \Omega$. (This reduces the problem to the simpler discrete time setting.)

2. Construct an internal martingale $M$ that solves the discrete time problem.

3. "Push $M$ down" to the required local martingale.

As the first step is the most technical, the proof of the main "lifting theorem" is deferred until §5. Some terminology from [H.P.] is required in order to state the result.

Terminology 2.2. An internal nondecreasing sequence of ${ }^{*}$-sub- $\sigma$-fields of $\mathbb{Q}^{1}$, $\left\{\mathscr{G}_{\underline{t}} \mid \underline{t} \in T\right\}$ is an internal filtration if $\mathscr{F}_{t}^{1}=\left(\cap_{o_{\underline{t}}>t} \sigma\left(\mathscr{M}_{\underline{t}}\right)\right) \vee \mathfrak{R}^{1}$. A $\mathscr{G}_{\underline{t}}$-stopping time is an internal mapping $V$ from $\Omega^{1}$ to $T \cup\{\infty\}$ such that $\{V=\underline{t}\} \in \mathscr{\mathscr { P }}_{\underline{t}}$ for all $\underline{t}$ in $T$. If ${ }^{\circ} V=U$ a.s., then $V$ is a $\mathscr{B}_{\underline{t}}$-lifting of $U$. In what follows, we consider a fixed internal filtration $\left\{G_{\underline{t}}\right\}$.

An internal stochastic process $X: T \times \Omega^{1} \rightarrow{ }^{*} \mathbf{R}^{d}$ (i.e. $X$ is internal and $X(\underline{t}, \cdot)$ is $\mathbb{Q}^{1}$-measurable for all $t$ in $\left.T\right)$ is of class $S D$ if for a.a. $\omega,{ }^{\circ} X(\underline{t}) \in \mathbf{R}^{d}$ for all $\underline{t}$ in $\operatorname{ns}(T)=\left\{\left.\underline{t} \in T\right|^{\circ} \underline{t}<\infty\right\}$, and

$$
\operatorname{st}(X)(t)=\lim _{0 \underline{t} \downarrow t}{ }^{\circ} X(\underline{t})
$$

defines a function, $\operatorname{st}(X)$, in $D\left(\mathbf{R}^{d}\right)$. We define st $(X)=0$ for $\omega$ such that the right side of (2.1) is not well defined for some $t \geqslant 0$. Note that if $X$ is $S D$ and $\Re_{\underline{t}}$-adapted

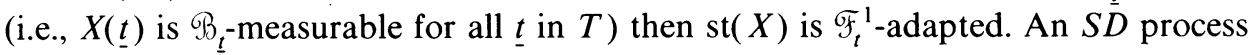
$X$ is of class $S D J$ if for a.a. $\omega, X(t) \approx X(0)$ for all $\underline{t} \approx 0$, and for each $t$ in $[0, \infty)$ there is a $\underline{t}(\omega) \approx t$ such that $X(\underline{u}) \approx \operatorname{st}(X)(t)$ if $\underline{u} \geqslant \underline{t}$ and $\underline{u} \approx t$, and $X(\underline{u}) \approx$ $\operatorname{st}(X)\left(t^{-}\right)$if $\underline{u}<t$ and $\underline{u} \approx t$. We say $X$ is an $S D$ (respectively, $S D J$ ) lifting of $x$ if $X$ is $S D$ (respectively, $S D J$ ) and $\operatorname{st}(X)(t)=x(t)$ for all $t \geqslant 0$ a.s. Note that these definitions include the case when $X(\underline{t}, \omega)=X(\underline{t})$ is deterministic.

A $\mathscr{G}_{\underline{t}}$-adapted $S D$ process $X: T \times \Omega \rightarrow{ }^{*} \mathbf{R}^{d}$ is $S$-locally integrable if there is a nondecreasing sequence of $\mathscr{P}_{\underline{t}}$-stopping times $\left\{V_{n}\right\}$ such that $\left\|X\left(V_{n} \wedge \underline{t}\right)\right\|$ is $S$ integrable (see Anderson [2, Definition 3]) for all $(\underline{t}, n)$ in $T \times \mathbf{N}, \lim _{n \rightarrow \infty}{ }^{\circ} V_{n}=\infty$ a.s., ${ }^{\circ} V_{n}<\infty$ a.s. and ${ }^{\circ} X\left(V_{n}\right)=\operatorname{st}(X)\left({ }^{\circ} V_{n}\right)$ a.s. The sequence $\left\{V_{n}\right\}$ is said to reduce $X$.

A $\mathscr{T}_{\underline{\underline{t}}}{ }^{*}$-increasing process is a $\mathscr{B}_{\underline{\underline{t}}}$-adapted process $A: T \times \Omega^{1} \rightarrow{ }^{*} \mathbf{R}$ of class $S D J$ such that for all $\omega, A(\cdot, \omega)$ is nondecreasing and $A(0, \omega)=0$. If $A$ is an $S D J$ lifting of $a$, we say $A$ is a $\mathscr{B}_{\underline{t}^{-}}$-increasing lifting of $a$.

A $\mathscr{P}_{\underline{t}}$-martingale is a $\mathscr{P}_{\underline{t}}$-adapted process $M: T \times \Omega^{1} \rightarrow{ }^{*} \mathbf{R}$ such that $\left\{\left(M(\underline{t}), \mathscr{R}_{\underline{t}}\right) \mid\right.$ $\underline{t} \in T\}$ is an internal martingale and $\sup _{(\underline{t}, \omega)}|M(\underline{t}, \omega)| \in{ }^{*} \mathbf{R}$. If, . . addition, $M$ is $S$-locally integrable then $M$ is an $S$-local martingale (with respect to $\left\{\Re_{\underline{t}}\right\}$ ). We say that $M$ is a $\mathscr{B}_{\underline{t}}$-local martingale lifting of $m$ if $M$ is an $S$-local martingale and an $S D J$ lifting of $m$.

Finally, if $X: T \times \Omega \rightarrow{ }^{*} \mathbf{R}$, let $\Delta(X)(\underline{t})=X(\underline{t}+\Delta t)-X(\underline{t})$. 
The correspondence between the above nonstandard definitions and their standard counterparts is studied in [H.P.] and will be used in §5. For now we only need the following simple result.

THEOREM 2.3 [H.P., THEOREM 5.2]. Let $\left\{9_{t}\right\}$ be an internal filtration.

(a) If $M$ is a $\Re_{t}$-martingale and $\left\{V_{n}\right\}$ is a sequence of $\mathscr{B}_{t}$-stopping times such that $\lim _{n \rightarrow \infty}{ }^{\circ} V_{n}=\infty$ a.s. and $\left|M\left(V_{n}\right)\right|$ is $S$-integrable, then $M$ is $S D$.

(b) If $M$ is an $S$-local martingale, then $\operatorname{st}(M)$ is an $\mathscr{F}_{t}^{1}$-local martingale.

We are ready to state the main lifting theorem.

THEOREM 2.4. There is an internal filtration $\left\{\mathscr{B}_{t}\right\}, a \mathscr{G}_{t}$-local martingale lifting of $n^{1}$, $N^{1}, a \Re_{t^{-}}-$-increasing lifting of $a^{1}, A^{1}$, and a sequence of $\mathscr{T}_{\underline{t}}^{-}{ }^{-}$-stopping times $\left\{W_{n}^{1}\right\}$ that satisfy the following conditions:

(2.2) Both $A^{1}$ and $N^{1}$ are reduced by $\left\{W_{n}^{1}\right\}$, and $A^{1}(\underline{t}+\Delta t)$ is $\mathscr{B}_{t}$-adapted.

(2.3) For a.a. $\omega$ if $\underline{t}_{1} \approx \underline{t}_{2} \in \mathrm{ns}(T),{ }^{\circ} \Delta N^{1}\left(\underline{t}_{1}\right) \neq 0$ and ${ }^{\circ} \Delta A^{1}\left(\underline{t}_{2}\right) \neq 0$ then $\underline{t}_{2} \leqslant \underline{t}_{1}$.

(2.4) There is a positive infinitesimal $\delta$ and an infinite natural number $\gamma$ such that $\delta \leqslant X^{1}(\underline{t}, \omega)=N^{1}(\underline{t}, \omega)+A^{1}(\underline{t}, \omega) \leqslant \gamma$ for all $(\underline{t}, \omega)$ in $T \times \Omega^{1}$.

Proof. See $§ 5$.

Note that $X^{1}$ need not be $S D J$ since $N^{1}$ and $A^{1}$ may have jumps at infinitely close, but distinct, times. Whenever this occurs, (2.3) implies that the jump in $A^{1}$ takes place first. As there were no additional conditions on the original internal filtration $\left\{\mathbb{Q}_{\underline{t}}^{1}\right\}$ we may, and shall, assume that $\mathscr{B}_{\underline{t}}=\mathbb{Q}_{\underline{t}}^{1}$.

We now enlarge our space as follows. Let $\left(\Omega^{2}, \mathbb{Q}^{2}, \bar{P}^{2}\right)=\left({ }^{*}[0,1]^{T}\right.$, $\left.* \mathscr{B}([0,1])^{T},{ }^{*} m^{T}\right)$, where $m$ is Lebesgue measure on $[0,1]$, and $\left({ }^{*}[0,1]^{T}, * \mathscr{B}([0,1])^{T}\right)$ denotes the internal product space. Let $\mathbb{Q}_{t}^{2}$ be the internal $\sigma$-algebra generated by the coordinate mappings on $\Omega^{2}$ up to and including $t$. Then define

$$
\left(\Omega, \mathbb{Q}, \bar{P}, \mathbb{Q}_{\underline{t}}\right)=\left(\Omega^{1} \times \Omega^{2}, \mathbb{Q}^{1} \times \mathbb{Q}^{2}, \bar{P}^{1} \times \bar{P}^{2}, \mathbb{Q}_{\underline{t}}^{1} \times \mathbb{Q}_{\underline{t}}^{2}\right)
$$

and let $\pi_{1}: \Omega \rightarrow \Omega_{1}$ be the projection map. The adapted Loeb space generated by $\left(\Omega, \mathscr{Q}, \bar{P}, \mathscr{Q}_{\underline{t}}\right)$ is denoted by $\left(\Omega, \mathscr{F}, P, \mathscr{F}_{t}\right)$. Integration with respect to $P^{1}$ and $P$ is denoted by $E^{1}$ and $E$, respectively.

LEMMA 2.5. (a) $\pi_{1}$ is measure-preserving and $\mathscr{F}_{s} \mid \mathscr{F}_{s}{ }^{1}$-measurable for all $s \geqslant 0$.

(b) If $\phi: \Omega^{1} \rightarrow[0, \infty)$ is $\mathscr{F}^{1}$-measurable then for all $s \geqslant 0, E\left(\phi \circ \pi_{1} \mid \mathscr{F}_{s}\right)=$ $E^{1}\left(\phi \mid \mathscr{F}_{s}^{1}\right) \circ \pi_{1}$ a.s.

Proof. (a) Since $\mathscr{F}_{1} \times \mathscr{F}_{2} \subset \mathscr{F}$ (see Anderson [2, Theorem 22]), $\pi_{1}$ is measurable and therefore is clearly measure-preserving. If $A \in \mathscr{F}_{s}^{1}$, then by Lemma 2.1 there is an $\underline{s} \approx s$ and a $B$ in $\mathbb{Q}_{\underline{s}}^{1}$ such that $P^{1}(A \Delta B)=0$. Therefore $P\left(\pi_{1}^{-1}(A) \Delta \pi_{1}^{-1}(B)\right)=0$ and hence $\pi_{1}^{-1}(A) \in \mathscr{\mathscr { F }}_{s}$ since $\pi_{1}^{-1}(B) \in \mathbb{Q}_{\underline{s}} \subset \mathscr{F}_{s}$.

(b) By truncating we may assume $\phi$ is bounded. Let $\Phi$ be a bounded $\mathbb{Q}^{1}$-measurable lifting of $\phi$ (see Loeb [9]). By Lemma 2.1 there exists $\underline{s} \approx s$ such that

$$
E\left(\phi \circ \pi_{1} \mid \mathscr{F}_{s}\right)=E\left(\phi \circ \pi_{1} \mid \sigma\left(\mathbb{Q}_{\underline{s}}\right)\right)={ }^{\circ} \bar{E}\left(\Phi \circ \pi_{1} \mid \mathbb{Q}_{\underline{s}}\right) \quad \text { a.s. }
$$


and

$$
E^{1}\left(\phi \mid \widetilde{F}_{s}^{1}\right) \circ \pi_{1}=E^{1}\left(\phi \mid \sigma\left(\mathbb{Q}_{\underline{s}}^{1}\right)\right) \circ \pi_{1}=\circ \bar{E}^{1}\left(\Phi \mid \mathbb{Q}_{\underline{s}}^{1}\right) \circ \pi_{1} \text { a.s. }
$$

The result follows since the right sides of the above equalities are clearly the same.

Let $x=x^{1} \circ \pi_{1}, n=n^{1} \circ \pi_{1}$ and $a=a^{1} \circ \pi_{1}$. The above lemma implies that $n$ is an $\mathscr{F}_{t}$-local martingale and that $\left(\left(x^{1}, a^{1}\right), \mathscr{F}_{.}^{1}\right)$ and $\left((x, a), \mathscr{F}_{.}\right)$are synonymous. In particular, by Aldous [1, Theorem 19.6] $a(t)$ is a predictable increasing process with respect to $\left\{\mathscr{F}_{t}\right\}$. It follows that $x(t)$ is a nonnegative local submartingale with respect to $\left\{\mathscr{F}_{t}\right\}$ and $x=n+a$ is its Doob-Meyer decomposition. We also define $A=A^{1} \circ \pi_{1}$, $N=N^{1} \circ \pi_{1}$ and $X=X^{1} \circ \pi_{1}$. Clearly $A, N$ and $X$ satisfy the same properties as $A^{1}$, $N^{1}$ and $X^{1}$, only with $\mathbb{Q}_{t}$ in place of $\mathbb{Q}_{t}^{1}$ and $W_{n}=W_{n}^{1} \circ \pi_{1}$ in place of $W_{n}^{1}$. Note also that $N(\underline{t})$ is an internal martingale with respect to the larger ${ }_{-\sigma}^{-\sigma}$-fields $\left\{\mathbb{Q}_{\underline{t}}^{\mathrm{i}} \times \mathbb{Q}^{2} \mid \underline{t}\right.$ $\in T\}$.

Fix $p \in[0,1]$, once and for all.

Notation 2.6. If $\omega \in \Omega$, we write $\omega=\left(\omega^{1}, \omega^{2}\right)=\left(\omega^{1},\left(\omega_{\underline{\underline{s}}}^{2}\right)_{\underline{\underline{s}} \in T}\right)$. Define $\phi: T \times \Omega \rightarrow$ $\{-1,1\}$ by

$$
\phi(0, \omega)= \begin{cases}1 & \text { if } \omega_{0}^{2} \leqslant p \\ -1 & \text { if } \omega_{0}^{2}>p\end{cases}
$$

and

$$
\phi(\underline{t}, \omega)= \begin{cases}-1 & \text { if } \omega_{\underline{t}}^{2} \leqslant \Delta A(\underline{t}-\Delta t, \omega)(2(X(\underline{t}-\Delta t, \omega)+\Delta A(\underline{t}-\Delta t, \omega)))^{-1}, \\ 1 & \text { otherwise. }\end{cases}
$$

Let $Z(\underline{t})=\prod_{\underline{s}=0}^{\underline{t}=0} \phi(\underline{s})$, and define $M(\underline{t})=Z(\underline{t}) X(\underline{t})$.

Hence $|M(\underline{t})|=X(\underline{t})$ and $M$ changes sign whenever $\phi=-1$. These added jumps are weighted to counteract the increasing part of $X$, and hence make $M$ an $\mathbb{Q}_{t}$-martingale.

LEMMA 2.7. $M$ is an $\mathbb{Q}_{\underline{t}}$-martingale.

Proof. It is clear from its definition that $M$ is an $\mathbb{Q}_{\underline{t}}$-adapted process and $|M(\underline{t})|=X(\underline{t}) \leqslant \gamma($ by $(2.4))$. Note that

$$
\begin{aligned}
\bar{E}\left(M(\underline{t}+\Delta t) \mid \mathbb{Q}_{\underline{t}}\right)=Z(\underline{t}) \bar{E}\left((X(\underline{t})+\Delta N(\underline{t})+\Delta A(\underline{t})) \phi(\underline{t}+\Delta t) \mid \mathbb{Q}_{\underline{t}}\right) \\
=Z(\underline{t})\left((X(\underline{t})+\Delta A(\underline{t})) \bar{E}\left(\phi(\underline{t}+\Delta t) \mid \mathbb{Q}_{\underline{t}}\right)\right. \\
\left.+\bar{E}\left(\Delta N(\underline{t}) \phi(\underline{t}+\Delta t) \mid \mathbb{Q}_{\underline{t}}\right)\right),
\end{aligned}
$$

because $A(\underline{t}+\Delta t)$ is $\mathbb{Q}_{\underline{t}}$-measurable. Since $N$ is an $\mathbb{Q}_{\underline{t}}^{1} \times \mathbb{Q}^{2}$-martingale and $\phi(\underline{t}+\Delta t)$ is $\mathbb{Q}_{\underline{t}}^{1} \times \mathbb{Q}^{2}$-measurable, the last term becomes

$$
\bar{E}\left(\phi(\underline{t}+\Delta t) \bar{E}\left(\Delta N(\underline{t}) \mid \mathbb{Q}_{\underline{t}}^{1} \times \mathbb{Q}^{2}\right) \mid \mathbb{Q}_{\underline{t}}\right)=0 .
$$

An easy computation now implies

$$
\begin{aligned}
\bar{E}\left(M(\underline{t}+\Delta t) \mid \mathbb{Q}_{\underline{t}}\right) & =Z(\underline{t})(X(\underline{t})+\Delta A(\underline{t}))\left(1-\Delta A(\underline{t})(X(\underline{t})+\Delta A(\underline{t}))^{-1}\right) \\
& =Z(\underline{t}) X(\underline{t})=M(\underline{t}) . \quad \square
\end{aligned}
$$


In order to apply Theorem 2.3 to obtain the required local martingale, we need the following lemma:

LEMMA 2.8. If $U=U^{1} \circ \pi_{1}$ for some $\mathscr{F}^{1}$-measurable random variable $U^{1}$, then

$$
P\left(\exists \underline{t} \in \mathrm{ns}(T) \ni \underline{t} \approx U, \quad{ }^{\circ} X(\underline{t})>0, \phi(\underline{t}+\Delta t)=-1 \text { and }^{\circ} \Delta A(\underline{t})=0\right)=0 .
$$

Proof. It clearly suffices to show that $P\left(B_{n, \varepsilon}\right)=0$ for each $n$ in $\mathbf{N}$ and positive infinitesimal $\varepsilon$, where

$$
\begin{aligned}
B_{n, \varepsilon}=\left\{\omega \mid \exists \underline{t} \leqslant n \text { such that }|\underline{t}-V|<\varepsilon, X(\underline{t}) \geqslant n^{-1},\right. \\
\phi(\underline{t}+\Delta t)=-1 \text { and } \Delta A(\underline{t}) \leqslant \varepsilon\}
\end{aligned}
$$

and $V=V^{1} \circ \pi_{1}$, for some $\mathbb{Q}^{1}$-measurable lifting of $U^{1}, V^{1}$. By Lemma 2.1 we have

$$
\begin{aligned}
P\left(B_{n, \varepsilon} \mid \sigma\left(\mathbb{Q}^{1} \times\left\{\phi, \Omega_{2}\right\}\right)\right)= & { }^{\circ} \bar{P}\left(B_{n, \varepsilon} \mid \mathbb{Q}^{1} \times\left\{\phi, \Omega_{2}\right\}\right) \quad \text { a.s. } \\
\leqslant & \sum_{\underline{t} \leqslant n} I_{\left\{|\underline{t}-\eta|<\varepsilon, \Delta A(\underline{t}) \leqslant \varepsilon, X(\underline{t}) \geqslant n^{-1}\right\}} \\
& \times \bar{P}\left(\phi(\underline{t}+\Delta t)=-1 \mid \mathbb{Q}^{1} \times\left\{\phi, \Omega_{2}\right\}\right) \\
\leqslant & \sum_{t \leqslant n} I_{\{\mid \underline{t}-\eta<\varepsilon, \Delta A(\underline{t}) \leqslant \varepsilon\}} \Delta A(\underline{t}) n / 2 \\
= & 0 \quad \text { a.s. }
\end{aligned}
$$

(the last line follows because $A$ is of class $S D J$ and $\varepsilon \approx 0$ ). The proof is completed by taking expectations in the above.

THEOREM 2.9. The process $M$ is $S D$ and $m=\operatorname{st}(M)$ is an $\left\{\mathscr{F}_{t}\right\}$-local martingale such that $|m|$ and $x$ are indistinguishable. Moreover, $\left((|m|, a), \mathscr{F}_{.}\right) \equiv\left(\left(x^{1}, a^{1}\right), \mathscr{F}_{.}^{1}\right)$, where $a$ and $a^{1}$ are the increasing processes in the Doob-Meyer decompositions of $|m|$ and $x^{1}$, respectively. If $x^{1}$ is a submartingale, $m$ is a martingale.

Proof. Since $\left|M\left(W_{n}\right)\right|=X\left(W_{n}\right)$ is $S$-integrable, $M$ is $S D$ by Theorem 2.3(a). We claim that $M$ is an $S$-local martingale. If ${ }^{\circ} M\left(W_{n}\right) \neq m\left({ }^{\circ} W_{n}\right) \quad(m \equiv \operatorname{st}(M))$, then, since ${ }^{\circ}\left|M\left(W_{n}\right)\right|=\left|m\left({ }^{\circ} W_{n}\right)\right|$ and ${ }^{\circ} A\left(W_{n}\right)=a\left({ }^{\circ} W_{n}\right)$ a.s., with probability one there is a positive infinitesimal $\underline{t}$ such that $\phi\left(W_{n}+\underline{t}\right)=-1$, ${ }^{\circ} X\left(W_{n}+\underline{t}-\Delta t\right)=x\left({ }^{\circ} W_{n}\right)>0$, and ${ }^{\circ} \Delta A\left(W_{n}+\underline{t}-\Delta t\right)=0$. Therefore

$$
\begin{aligned}
\left\{{ }^{\circ} M\left(W_{n}\right) \neq m\left({ }^{\circ} W_{n}\right)\right\} \subset\{\exists \underline{t} \in \mathrm{ns}(T) \ni \underline{t} \approx & \approx W_{n},{ }^{\circ} X(\underline{t})>0, \\
\phi(\underline{t}+\Delta t) & \left.=-1 \text { and }{ }^{\circ} \Delta A(\underline{t})=0\right\} \cup \Lambda
\end{aligned}
$$

for some null set $\Lambda$. The previous lemma implies that ${ }^{\circ} M\left(W_{n}\right)=m\left({ }^{\circ} W_{n}\right)$ a.s. and hence proves the claim. By Theorem 2.3(b), $m$ is an $\left\{\mathscr{F}_{t}\right\}$-local martingale, and

$$
|m|=|\operatorname{st}(M)|=\operatorname{st}(|M|)=x \quad \text { a.s. }
$$

Lemma 2.5 implies that $\left((|m|, a), \mathscr{F}_{.}\right) \equiv\left(\left(x^{1}, a^{1}\right), \mathscr{F}_{.}^{1}\right)$. If $x^{1}$ is a submartingale, then so is $|m|=x$. From this and the fact that $m$ is a local martingale, it follows easily that $m$ must be a martingale. 
3. The distribution of $m$. In this section we describe the finite-dimensional distributions of $m$ in terms of the law of $(x, a)$. In order to facilitate the computations we will modify the definition of $m$ by adding a positive infinitesimal to the lifting $N$ of $n$ obtained in Theorem 2.4 (see Remark 3.8). A series of technical lemmas precedes the proof of the main result (Theorem 3.11).

Notation 3.1. Let $\mathcal{C}$ denote the internal $\sigma$-algebra of internal subsets of $T$. If $F$ : $T \rightarrow{ }^{*} \mathbf{R}$ is an internal function, let $\nu_{F}$ denote the internal signed measure on $(T, \mathcal{C})$ defined by $\nu_{F}(\{\underline{t}\})=F(\underline{t}+\Delta t)-F(\underline{t})$. If $F: T \times \Omega \rightarrow{ }^{*} \mathbf{R}$ is an internal stochastic process, then $\nu_{F}$ denotes the internal random signed measure defined by the above.

The following two elementary results [H.P., Lemma 2.7 and Theorem 4.7(b)] are frequently used.

Lemma 3.2. If $F: T \rightarrow{ }^{*} \mathbf{R}$ is an internal, nondecreasing function of class $S D$ such that $F(\underline{t}) \approx F(0)$ for all $\underline{t} \approx 0$, and $f=\operatorname{st}(F)$, then for each $B$ in $\Re([0, \infty))$, $L\left(\nu_{F}\right)\left(\left\{\left.\underline{t} \in T\right|^{\circ} \underline{t} \in B\right\}\right)=\int_{B} d f$.

Lemma 3.3. If $X: T \times \Omega \rightarrow{ }^{*} \mathbf{R}^{d}$ is an $S D$ internal stochastic process then for each $t \geqslant 0$ there is $a \underline{t}^{1} \approx t$ such that for almost all $\omega$ if $t \approx t$ and $\underline{t} \geqslant \underline{t}^{1}$ then ${ }^{\circ} X(\underline{t})=\operatorname{st}(X)(t)$.

To obtain a formula for the distribution of $m$, it is clear we need to examine $\operatorname{sgn}(m(t))$. The following result allows us to study $\operatorname{sgn}(m(t))$ by means of the internal process $Z(t)$.

Lemma 3.4. For each $t \geqslant 0$ there is a $t^{1} \approx t$ such that with probability one, $m(t)=Z(\underline{t}) x(t)$ for all $\underline{t} \geqslant \underline{t}^{1}$ that satisfy $\underline{t} \approx t$. If $t=0$, we may take $\underline{t}^{1}=0$.

Proof. Since $(M, X)$ is an $S D$ lifting of $(m, x)$, by Lemma 3.3 there is a $t^{1} \approx t$ such that ${ }^{\circ}(M(\underline{t}), X(\underline{t}))=(m(t), x(t))$ for all $\underline{t} \geqslant \underline{t}^{1}$ satisfying $\underline{t} \approx t$ and a.a. $\omega$. Therefore for a.a. $\omega$ if $\underline{t} \geqslant \underline{t}^{1}$ and $\underline{t} \approx t$, then

$$
m(t)={ }^{\circ} M(\underline{t})=Z(\underline{t})^{\circ} X(\underline{t})=Z(\underline{t}) x(t) .
$$

If $t=0$ and $x(0)=0$, then $m(0)=Z(0) x(0)=0$ a.s. If $t=0$ and $x(0)>0$, then ${ }^{\circ} X(\underline{t})>0$ for all $t \approx 0$ a.s. and therefore, by Lemma 2.7 (with $U=0$ ), $\phi(\underline{t}+\Delta t)=1$ for all infinitesimal $t$ in $T$ a.s. It follows that for a.a. $\omega$ and for all $t \approx 0$,

$$
m(0)=Z(\underline{t}) x(0)=Z(0) x(0) .
$$

LEMMA 3.5 .

$$
L\left(\nu_{A}\right)\left(\left\{\left.\underline{t} \in \mathrm{ns}(T)\right|^{\circ} X(\underline{t}) \neq x\left({ }^{\circ} \underline{t}^{-}\right) \text {or }{ }^{\circ} \Delta A(\underline{t}) \neq \Delta a\left({ }^{\circ} \underline{t}\right)\right\}\right)=0 \text { a.s. }
$$

Proof. Let $\left\{V_{i} \mid i \in \mathbf{N}\right\}$ be a sequence of $\mathbb{Q}_{\underline{t}}$-stopping times such that

$$
\left\{\left.\underline{t} \in \mathrm{ns}(T)\right|^{\circ}|X(\underline{t})-X(\underline{t}-\Delta t)|>0\right\}=\left\{\left.V_{i}\right|^{\circ} V_{i} \neq \infty\right\} \quad \text { a.s. }
$$


(such a sequence clearly exists since $X$ is the sum of two $S D J$ processes). Therefore,

$$
\left\{\left.\underline{t} \in \operatorname{ns}(T)\right|^{\circ} X(\underline{t}) \neq x\left({ }^{\circ} \underline{t}^{-}\right)\right\} \subset \bigcup_{i \in \mathbf{N}} \bigcap_{m \in \mathbf{N}}\left(\left[V_{i}, V_{i}+m^{-1}\right) \cap T\right) \text { a.s. }
$$

Moreover by (2.3) we have ${ }^{\circ} A\left(V_{i}\right)=a\left({ }^{\circ} V_{i}\right)$ a.s. and, hence,

$$
\begin{aligned}
L\left(\nu_{A}\right)\left(\bigcup_{i \in \mathbf{N}} \bigcap_{m \in \mathbf{N}}(\right. & {\left.\left.\left[V_{i}, V_{i}+m^{-1}\right) \cap T\right)\right) } \\
& \leqslant \sum_{i=1}^{\infty} \lim _{m \rightarrow \infty} L\left(\nu_{A}\right)\left(\left[V_{i}, V_{i}+m^{-1}\right) \cap T\right)=0 \text { a.s. }
\end{aligned}
$$

Thus it follows that $L\left(\nu_{A}\right)\left(\left\{\left.\underline{t} \in \mathrm{ns}(T)\right|^{\circ} X(\underline{t}) \neq x\left({ }^{\circ} \underline{t}^{-}\right)\right\}\right)=0$ a.s. A similar argument shows that $L\left(\nu_{A}\right)\left(\left\{\left.\underline{t} \in \mathrm{ns}(T)\right|^{\circ} \Delta A(\underline{t}) \neq \Delta a\left({ }^{\circ} \underline{t}\right)\right\}\right)=0$ a.s., and the proof is complete.

Let

$$
a^{d}(t)=\int_{0}^{t} I(\Delta a(s)>0) d a(s) \text { and } a^{c}(t)=\int_{0}^{t} I(\Delta a(s)=0) d a(s)
$$

be the pure jump and continuous parts of $a$, respectively. A corresponding decomposition of $A$ is given by the following:

LEMMA 3.6. For some $H$ in $* \mathbf{N}-\mathbf{N}, A^{c}(\underline{t})=\sum_{\underline{s}<\underline{t}} \Delta A(\underline{s}) I\left(\Delta A(\underline{s})<H^{-1}\right)$ and $A^{d}=A-A^{c}$ are SDJ liftings of $a^{c}$ and $a^{d}$, respectively.

The simple proof is left for the reader. Fix $H$ and define $A^{c}$ and $A^{d}$ as in the above. Let $\left\{r_{i} \mid i \in \mathbf{N}\right\}$ be an enumeration of the nonnegative rationals and choose $\underline{r}_{i} \approx r_{i}$ $\left(\underline{r}_{i} \in T\right)$ such that $m\left(r_{i}\right)=Z\left(\underline{r}_{i}\right) x\left(r_{i}\right)$ and ${ }^{\circ} A\left(\underline{t}_{i}\right)=a\left(r_{i}\right)$ a.s. The existence of $\underline{r}_{i}$ follows from Lemmas 3.3 and 3.4. Since $A(0)=a(0)=0$ we may let $\underline{r}_{i}=0$ if $r_{i}=0$.

LEMMA 3.7. There is a $K$ in ${ }^{*} \mathbf{N}-\mathbf{N}$ such that $K H^{-1} \approx 0$, and for all $(i, j)$ with $r_{i}<r_{j}$

$$
\begin{aligned}
\circ \int I\left(\underline{r}_{i} \leqslant \underline{s}<\underline{r}_{j}\right)\left(K^{-1}+X(\underline{s})+\Delta A(\underline{s})\right)^{-1} d \nu_{A^{c}} \\
=\int I\left(r_{i}<s \leqslant r_{j}\right) x(s)^{-1} d a^{c}(s) \text { a.s. }
\end{aligned}
$$

and

$$
\begin{aligned}
\circ \int I\left(\underline{r}_{i} \leqslant \underline{s}<\underline{r}_{j}\right) & \left(K^{-1}+X(\underline{s})+\Delta A(\underline{s})\right)^{-1} d \nu_{A^{d}} \\
& =\int I\left(r_{i}<s \leqslant r_{j}\right)\left(x\left(s^{-}\right)+\Delta a(s)\right)^{-1} d a^{d}(s) \text { a.s. }
\end{aligned}
$$


Proof. If $r_{i}<r_{j}$ we claim that (3.1) and (3.2) hold for $K$ in N. Indeed if $K \in \mathbf{N}$,

$$
\begin{aligned}
& \circ I\left(\underline{r}_{i} \leqslant \underline{s}<\underline{r}_{j}\right)\left(K^{-1}+X(\underline{s})+\Delta A(\underline{s})\right)^{-1} d v_{A^{d}} \\
& =\int I\left(\underline{r}_{i} \leqslant \underline{s}<\underline{r}_{j}\right)\left(K^{-1}+x\left({ }^{\circ} \underline{s}^{-}\right)+\Delta a\left({ }^{\circ} \underline{s}\right)\right)^{-1} d L\left(\nu_{A^{d}}\right) \text { a.s. } \\
& =\int I\left(r_{i}<{ }^{\circ} \underline{s} \leqslant r_{j}\right)\left(K^{-1}+x\left({ }^{\circ} \underline{s}^{-}\right)+\Delta a\left({ }^{\circ} \underline{s}\right)\right)^{-1} d L\left(\nu_{A^{d}}\right) \text { a.s. } \\
& \text { ( since }{ }^{\circ} A\left(\underline{r}_{k}\right)=a\left(r_{k}\right) \text { a.s.) } \\
& =\int I\left(r_{i}<s \leqslant r_{j}\right)\left(K^{-1}+x\left(s^{-}\right)+\Delta a(s)\right)^{-1} d a^{d}(s) \text { a.s. }
\end{aligned}
$$

(Lemmas 3.2 and 3.6).

The same result holds with $A^{c}$ and $a^{c}$ in place of $A^{d}$ and $a^{d}$, and hence the claim is proved. Let

$$
\begin{aligned}
& X_{i, j, K}^{c}=\arctan \left(\int I\left(\underline{r}_{i} \leqslant \underline{s}<\underline{r}_{j}\right)\left(K^{-1}+X(\underline{s})+\Delta A(\underline{s})\right)^{-1} d \nu_{A^{c}}\right), \\
& X_{i, j, K}^{d}=\arctan \left(\int I\left(\underline{r}_{i} \leqslant \underline{s}<\underline{r}_{j}\right)\left(K^{-1}+X(\underline{s})+\Delta A(\underline{s})\right)^{-1} d \nu_{A^{d}}\right),
\end{aligned}
$$

and let $X_{i, j}^{c}$ and $X_{i, j}^{d}$ be uniformly bounded liftings of

$$
\begin{array}{r}
\arctan \left(\int I\left(r_{i}<s \leqslant r_{j}\right) x(s)^{-1} d a^{c}(s)\right) \text { and } \\
\arctan \left(\int I\left(r_{i}<s \leqslant r_{j}\right)\left(x\left(s^{-}\right)+\Delta a(s)\right)^{-1} d a^{d}(s)\right),
\end{array}
$$

respectively. Since (3.1) and (3.2) hold for $K$ in $\mathbf{N}$, the monotone convergence theorem implies that

$$
\lim _{K \rightarrow \infty}{ }^{\circ} \bar{E}\left(\left|X_{i, j, K}^{c}-X_{i, j}^{c}\right|+\left|X_{i, j, K}^{d}-X_{i, j}^{d}\right|\right)=0,
$$

whenever $r_{i}<r_{j}$. Therefore for each $n$ in $\mathbf{N}$ there is a $K_{n}$ in ${ }^{*} \mathbf{N}-\mathbf{N}$ such that whenever $K \in{ }^{*} \mathbf{N}-\mathbf{N}$ satisfies $K \leqslant K_{n}$,

$$
\max _{\substack{i, j \leq n \\ r_{i}<r_{j}}} \bar{E}\left(\left|X_{i, j, K}^{c}-X_{i, j}^{c}\right|+\left|X_{i, j, K}^{d}-X_{i, j}^{d}\right|\right) \leqslant 2^{-n} .
$$

Extend $\left\{K_{n} \mid n \in \mathbf{N}\right\}$ internally to ${ }^{*} \mathbf{N}$ such that $K_{n} \geqslant n$. Let $K=\left(\min _{n \leqslant \gamma} K_{n}\right) \wedge H^{1 / 2}$ for some $\gamma$ in ${ }^{*} \mathbf{N}-\mathbf{N}$. Then $K$ is the required number.

Remark 3.8. If $N^{1}$ and $X^{1}$ are replaced by $N^{1}+K^{-1}$ and $X^{1}+K^{-1}$, respectively ( $A^{1}$ remains unchanged), then the conditions of Theorem 2.4 remain satisfied. Thus we may, and shall, assume that

$$
X(\underline{t})^{-1} H^{-1} \approx 0 \text { for all }(\underline{t}, \omega)
$$




$$
\begin{aligned}
\circ \int I\left(\underline{r}_{i} \leqslant \underline{s}<\underline{r}_{j}\right)(X(s)+\Delta A(\underline{s}))^{-1} d \nu_{A^{c}} \\
\quad=\int I\left(r_{i}<s \leqslant r_{j}\right) x(s)^{-1} d a^{c}(s) \quad \text { for all } r_{i}<r_{j} \text { a.s. }
\end{aligned}
$$

and

$$
\begin{aligned}
\circ \int I\left(\underline{r}_{i} \leqslant \underline{s}<\underline{r}_{j}\right)(X(\underline{s})+\Delta A(\underline{s}))^{-1} d \nu_{A^{d}} \\
\quad=\int I\left(r_{i}<s \leqslant r_{j}\right)\left(x\left(s^{-}\right)+\Delta a(s)\right)^{-1} d a^{d}(s) \text { for all } r_{i}<r_{j} \text { a.s. }
\end{aligned}
$$

LEMMA 3.9. Let $F: T \rightarrow{ }^{*} \mathbf{R}$ be an SDJ nondecreasing internal function, $f=\operatorname{st}(F)$ and let $\underline{t}_{1}<\underline{t}_{2} \mathrm{~ns}(T)$ such that for $i=1,2,{ }^{\circ} F\left(\underline{t}_{i}\right)=f\left({ }^{\circ} \underline{t}_{i}\right)$. If $G: T \rightarrow{ }^{*}[0, \infty)$ and $g$ : $[0, \infty) \rightarrow[0, \infty)$ satisfy ${ }^{\circ} G(\underline{s})=g\left({ }^{\circ} \underline{s}\right), L\left(\nu_{F}\right)$-a.s. and

$$
\circ I\left(\underline{t}_{1} \leqslant \underline{s}<\underline{t}_{2}\right) G(\underline{s}) d \nu_{F}=\int I\left({ }^{\circ} \underline{t}_{1}<s \leqslant{ }^{\circ} \underline{t}_{2}\right) g(s) d f(s)<\infty
$$

then $H(\underline{t})=\int I\left(\underline{t}_{1} \leqslant \underline{s}<\underline{t} \wedge \underline{t}_{2}\right) G(\underline{s}) d \nu_{F}$ is an SDJ lifting of

$$
h(t)=\int I\left({ }^{\circ} \underline{t}_{1}<s \leqslant t \wedge{ }^{\circ} \underline{t}_{2}\right) g(s) d f(s) .
$$

Proof. If $\underline{t}_{1} \leqslant \underline{t} \leqslant t_{2}$ and ${ }^{\circ} F(\underline{t})=f\left({ }^{\circ} \underline{t}\right)$, then

$$
\begin{aligned}
\circ \int I\left(\underline{t}_{1} \leqslant \underline{s}<\underline{t}\right) G(\underline{s}) d \nu_{F} & \geqslant \int I\left(\underline{t}_{1} \leqslant \underline{s}<\underline{t}\right) g\left({ }^{\circ} \underline{s}\right) L\left(\nu_{F}\right) \\
& =\int I\left({ }^{\circ} \underline{t}_{1}<{ }^{\circ} \underline{s} \leqslant{ }^{\circ} \underline{t}\right) g\left({ }^{\circ} \underline{s}\right) L\left(\nu_{F}\right) \\
& =\int I\left({ }^{\circ} \underline{t}_{1}<s \leqslant{ }^{\circ} \underline{t}\right) g(s) d f(s) \quad \text { (Lemma 3.2). }
\end{aligned}
$$

A similar argument shows that

$$
\circ I\left(\underline{t} \leqslant \underline{s}<\underline{t}_{2}\right) G(\underline{s}) d \nu_{F} \geqslant \int I\left({ }^{\circ} \underline{t}<s \leqslant{ }^{\circ} \underline{t}_{2}\right) g(s) d f(s) .
$$

Since the sum of the above inequalities leads to an equality, it follows that ${ }^{\circ} H(\underline{t})=h\left({ }^{\circ} \underline{t}\right)$. Similarly, if ${ }^{\circ} F(\underline{t})=f\left({ }^{\circ} \underline{t}^{-}\right)$and $\underline{t}_{1}<\underline{t} \leqslant \underline{t}_{2}$, then ${ }^{\circ} H(\underline{t})=h\left({ }^{\circ} \underline{t}^{-}\right)$. Note also that if $\underline{t} \geqslant \underline{t}_{2}$ or $\underline{t} \leqslant \underline{t}_{1}$ then ${ }^{\circ} H(\underline{t})=h\left({ }^{\circ} t\right)$. The result follows because $F$ is $S D J$ and $f=\operatorname{st}(F)$.

Lemma 3.10. Suppose $f$ is a nondecreasing pure jump function in $D(\mathbf{R})$, (i.e., $\left.f(t)=\sum_{s \leqslant t} \Delta f(s)\right)$ and $F: T \rightarrow{ }^{*} \mathbf{R} \cdot$ is a nondecreasing SDJ lifting of $f$. Let $h$ : $[0, \infty) \rightarrow \mathbf{R}$ be Lipschitz continuous and satisfy $h(0)=0$. If $g(t)=\sum_{s \leqslant t} h(\Delta f(s))$ and $G(\underline{t})=\Sigma_{\underline{s}<\underline{t}} h(\Delta F(\underline{s}))$, then ${ }^{\circ} F(\underline{t})=f\left({ }^{\circ} \underline{t}\right)$ implies ${ }^{\circ} G(\underline{t})=g\left({ }^{\circ} \underline{t}\right)$. 
Proof. Fix $t \geqslant 0$ and $\underline{t} \approx t$ such that ${ }^{\circ} F(t)=f(t)$. Let $\left\{t_{i} \mid i \in I\right\}$ $=\{s \leqslant t \mid \Delta f(s)>0\}$ and for each $t_{i}$ choose $\underline{t}_{i} \approx t_{i}$ such that ${ }^{\circ} \Delta F\left(\underline{t}_{i}\right)=\Delta f\left(t_{i}\right)$. Since ${ }^{\circ} F(\underline{t})=f\left({ }^{\circ}\right)$, it follows that $\left\{\underline{t}_{i} \mid i \in I\right\} \subset[0, \underline{t})$. If $|h(x)| \leqslant c|x|$, then

$$
\begin{aligned}
\limsup _{n \rightarrow \infty}{ }^{\circ} \sum I\left(\underline{s} \notin\left\{\underline{\underline{s}} \underline{\underline{t}}, \ldots, \underline{t}_{n}\right\}\right)|h(\Delta F(\underline{s}))| \\
\quad \leqslant c \limsup _{n \rightarrow \infty}{ }^{\circ} \sum_{\underline{s}<\underline{t}} I\left(\underline{s} \notin\left\{\underline{t}_{1}, \ldots, \underline{t}_{n}\right\}\right) \Delta F(\underline{s}) \\
\quad=c \limsup _{n \rightarrow \infty}\left(f(t)-\sum_{i=1}^{n} \Delta f\left(t_{i}\right)\right)=0 .
\end{aligned}
$$

Therefore

$$
{ }^{\circ} G(\underline{t})=\lim _{n \rightarrow \infty} \sum_{i=1}^{n} h\left(\Delta F\left(\underline{t}_{i}\right)\right)=\lim _{n \rightarrow \infty} \sum_{i=1}^{n} h\left(\Delta f\left(t_{i}\right)\right)=g(t) .
$$

We are finally ready for the main result of this section.

TheOrem 3.11. Suppose $\left\{B_{0}, \ldots, B_{n}\right\} \subset \Re((0, \infty)),\left\{e_{1}, \ldots, e_{n}\right\} \subset\{-1,1\}, e_{0} \in$ $\{-1,0,1\}$, and $0=t_{0}<t_{1}<\cdots<t_{n}$. Then $E(m(0) \mid a, x)=(2 p-1) x(0)$ a.s. and

$$
\begin{aligned}
& P\left(\left(m\left(t_{0}\right), \ldots, m\left(t_{n}\right)\right) \in e_{0} B_{0} \times \cdots \times e_{n} B_{n}\right) \\
& =\left(p I\left(e_{0}=1\right)+(1-p) I\left(e_{0}=-1\right)+I\left(e_{0}=0\right)\right) \\
& \times E\left(I\left(\left(x\left(t_{0}\right), \ldots, x\left(t_{n}\right)\right) \in\left|e_{0}\right| B_{0} \times B_{1} \times \cdots \times B_{n}\right)\right. \\
& \quad \times 2^{-n} \prod_{i=1}^{n}\left(1+\hat{e}_{i-1} \hat{e}_{i} \exp \left\{-\int_{t_{i}, 1}^{t_{t}} x(s)^{-1} d a^{c}(s)\right\}\right. \\
& \left.\left.\times \prod_{t_{i-1}<s \leqslant t_{i}}\left(1-\left(x\left(s^{-}\right)+\Delta a(s)\right)^{-1} \Delta a(s)\right)\right)\right),
\end{aligned}
$$

where

$$
\hat{e}_{i}= \begin{cases}e_{i} & \text { if } i>0 \text { or } e_{i} \neq 0, \\ 2 p-1 & \text { if } i=0 \text { and } e_{0}=0 .\end{cases}
$$

ProOF. By a routine limiting argument it suffices to prove the result when $t_{1}, \ldots, t_{n}$ are rationals. By the definition of $\left\{\underline{r}_{i}\right\}$, and (3.4) and (3.5), there are points $0=\underline{t}_{0}<\cdots<\underline{t}_{n}$ such that ${ }^{\circ} \underline{t}_{i}=t_{i}, m\left(t_{i}\right)=Z\left(\underline{t}_{i}\right) x\left(t_{i}\right)$ a.s., ${ }^{\circ} A\left(\underline{t}_{i}\right)=a\left(t_{i}\right)$ a.s.,

$$
\begin{aligned}
\circ \int I\left(\underline{t}_{i} \leqslant \underline{s}<\underline{t}_{i+1}\right)(X(\underline{s})+\Delta A(\underline{s}))^{-1} d \nu_{A^{c}} \\
=\int I\left(t_{i}<s \leqslant t_{i+1}\right) x(s)^{-1} d a^{c}(s) \text { a.s., }
\end{aligned}
$$


and

$$
\begin{aligned}
\circ \int I\left(\underline{t}_{i} \leqslant \underline{s}<\underline{t}_{i+1}\right)(X(\underline{s})+\Delta A(\underline{s}))^{-1} d \nu_{A^{d}} \\
\quad=\int I\left(t_{i}<s \leqslant t_{i+1}\right)\left(x\left(s^{-}\right)+\Delta a(s)\right)^{-1} d a^{d}(s) \text { a.s. }
\end{aligned}
$$

Then

$$
\begin{array}{r}
P\left(\left(m\left(t_{0}\right), \ldots, m\left(t_{n}\right)\right) \in e_{0} B_{0} \times \cdots \times e_{n} B_{n}\right) \\
=E\left(I\left(\left(Z(0) x(0), x\left(t_{1}\right), \ldots, x\left(t_{n}\right)\right) \in e_{0} B_{0} \times B_{1} \times \cdots \times B_{n}\right)\right. \\
\times I\left(\left(Z\left(\underline{t}_{1}\right), \ldots, Z\left(\underline{t}_{n-1}\right)\right)=\left(e_{1}, \ldots, e_{n-1}\right)\right) \\
\left.\quad \times P\left(Z\left(\underline{t}_{n}\right)=e_{n} \mid L\left(\mathbb{Q}^{1} \times \mathbb{Q}_{t_{n-1}}^{2}\right)\right)\right) .
\end{array}
$$

We claim that

$$
\begin{aligned}
& P\left(Z\left(\underline{t}_{n}\right)=e_{n} \mid L\left(\mathbb{Q}^{1} \times \mathbb{Q}_{\underline{t}_{n-1}}^{2}\right)\right) \\
&=\frac{1}{2}\left(1+e_{n} Z\left(\underline{t}_{n-1}\right) \exp \left\{-\int_{t_{n-1}}^{t_{n}} x(s)^{-1} d a^{c}(s)\right\}\right. \\
&\left.\quad \times \prod_{t_{n-1}<s \leqslant t_{n}}\left(1-\left(x\left(s^{-}\right)+\Delta a(s)\right)^{-1} \Delta a(s)\right)\right) .
\end{aligned}
$$

The definition of $Z$ implies that

$$
\begin{aligned}
\bar{E}\left(Z\left(\underline{t}_{n}\right) \mid \mathbb{Q}^{1} \times \mathbb{Q}_{\underline{t}_{n-1}}^{2}\right) & =Z\left(\underline{t}_{n-1}\right) \bar{E}\left(\prod_{\underline{t}_{n-1}<\underline{s} \leq \underline{t}_{n}} \phi(\underline{s}) \mid \mathbb{Q}^{1} \times \mathbb{Q}_{\underline{t}_{n-1}}^{2}\right) \\
& =Z\left(\underline{t}_{n-1}\right) \prod_{\underline{t}_{n-1} \leq \underline{s}<\underline{t}_{n}}\left(1-(X(\underline{s})+\Delta A(\underline{s}))^{-1} \Delta A(\underline{s})\right),
\end{aligned}
$$

where we have used the fact that $\left\{\phi(\underline{s}) \mid \underline{t}_{n-1}<\underline{s} \leqslant \underline{t}_{n}\right\}$ are conditionally independent given $\mathbb{Q}^{1} \times \mathbb{Q}_{\underline{t}_{n-1}}^{2}$.

To prove (3.10), assume first that

$$
\int\left(t_{n-1}<s \leqslant t_{n}\right)\left(x\left(s^{-}\right)+\Delta a(s)\right)^{-1} d a(s)=\infty .
$$

Then (3.7) and (3.8) imply that for a.a. $\omega$,

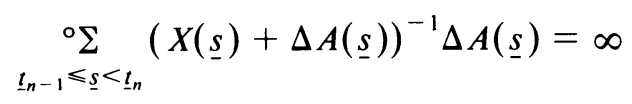

and, therefore by $(3.11),{ }^{\circ} \bar{E}\left(Z\left(\underline{t}_{n}\right) \mid \mathbb{Q}^{1} \times \mathbb{Q}_{\underline{t}_{n-1}}^{2}\right)=0$ a.s. This implies that

$$
P\left(Z\left(\underline{t}_{n}\right)=e_{n} \mid L\left(\mathbb{Q}^{1} \times \mathbb{Q}_{\underline{t}_{n-1}}^{2}\right)\right)={ }^{\circ} \bar{P}\left(Z\left(\underline{t}_{n}\right)=e_{n} \mid \mathbb{Q}^{1} \times \mathbb{Q}_{\underline{t}_{n-1}}^{2}\right) \text { a.s. }
$$

(Lemma 2.1)

$$
=1 / 2 \text { a.s. }
$$

Clearly the right side of (3.10) is also $\frac{1}{2}$ by assumption, and the claim is proved in this case. 
Assume now that

$$
\int I\left(t_{n-1}<s \leqslant t_{n}\right)\left(x\left(s^{-}\right)+\Delta a(s)\right)^{-1} d a(s)<\infty .
$$

If ${ }^{\circ}(X(\underline{s})+\Delta A(\underline{s}))^{-1} \Delta A(\underline{s})=1$ for some $\underline{s}$ in $\left[\underline{t}_{n-1}, \underline{t}_{n}\right)$, then by $(3.11)$, ${ }^{\circ} \bar{E}\left(Z\left(\underline{t}_{n}\right) \mid \mathbb{Q}^{1} \times \mathbb{Q}_{\underline{t}_{n-1}}^{\overline{2}^{2}}\right)=0$ a.s. and therefore $P\left(\bar{Z}\left(\underline{t}_{n}\right)=e_{n} \mid L\left(\mathbb{Q}^{1} \times \mathbb{Q}_{\underline{t}_{n-1}}^{2}\right)\right)=\frac{1}{2}$ a.s., as before. On the other hand, (3.7), (3.8) and Lemma 3.9 imply that for a.a. $\omega$, ${ }^{\circ} \underline{s}>t_{n-1}$ and $\left(x\left({ }^{\circ} \underline{s}^{-}\right)+\Delta a\left({ }^{\circ} \underline{s}\right)\right)^{-1} \Delta a\left({ }^{\circ} \underline{s}\right)=1$. This implies that the right side of (3.10) is also $\frac{1}{2}$. Hence, we may assume that

$$
{\stackrel{\circ}{t_{n-1}} \leq s<t_{n}}_{\max }(X(\underline{s})+\Delta A(\underline{s}))^{-1} \Delta A(\underline{s})=y(\omega)<1 .
$$

From (3.11) we obtain

$$
\begin{aligned}
\log \left(\bar{E}\left(Z\left(\underline{t}_{n}\right) / Z\left(\underline{t}_{n-1}\right) \mid \mathbb{Q}^{1} \times \mathbb{Q}_{\underline{t}_{n-1}}^{2}\right)\right) \\
=\sum_{\underline{t}_{n-1} \leqslant \underline{s}<\underline{t}_{n}} \log \left(1-(X(\underline{s})+\Delta A(\underline{s}))^{-1} \Delta A^{c}(\underline{s})\right) \\
\quad+\sum_{\underline{t}_{n-1} \leqslant \underline{s}^{<} \underline{t}_{n}} \log \left(1-(X(\underline{s})+\Delta A(\underline{s}))^{-1} \Delta A^{d}(\underline{s})\right) \\
=\sum_{1}+\sum_{2} .
\end{aligned}
$$

By (3.7), (3.8) and Lemma 3.9 we see that for a.a. $\omega$ satisfying the above assumptions, the hypotheses of Lemma 3.10 are satisfied, where

$$
\begin{aligned}
h(x) & =\log (1-(x \vee(-y(\omega))) \wedge y(\omega)), \\
f(t) & =\int I\left(t_{n-1}<s \leqslant t_{n} \wedge t\right)\left(x\left(s^{-}\right)+\Delta a(s)\right)^{-1} d a^{d}(s), \\
F(\underline{t}) & =\int I\left(\underline{t}_{n-1} \leqslant \underline{s}<\underline{t}_{n} \wedge \underline{t}\right)(X(\underline{s})+\Delta A(\underline{s}))^{-1} d \nu_{A^{d}} .
\end{aligned}
$$

Therefore Lemma 3.10 implies that for a.a. $\omega$ satisfying the above conditions,

$$
\begin{aligned}
\circ \sum_{2} & =\sum_{\underline{t}_{n-1} \leqslant \underline{s}<\underline{t}_{n}} h\left((X(\underline{s})+\Delta A(\underline{s}))^{-1} \Delta A^{d}(\underline{s})\right) \\
& =\sum_{t_{n-1}<s \leqslant t_{n}} \log \left(1-\left(x\left(s^{-}\right)+\Delta a(s)\right)^{-1} \Delta a(s)\right) .
\end{aligned}
$$

For $\Sigma_{1}$, let $\log (1-z)=-z-z^{2} \theta(z)$ for $|z|<1$ and note that

$$
\begin{aligned}
\circ \sum_{1}=- & \circ \sum_{\underline{t}_{n-1} \leq \underline{s}<\underline{t}_{n}}(X(\underline{s})+\Delta A(\underline{s}))^{-1} \Delta A^{c}(\underline{s}) \\
& -\sum_{\underline{t}_{n-1} \leq \underline{s}<\underline{t}_{n}}(X(\underline{s})+\Delta A(\underline{s}))^{-2}\left(\Delta A^{c}(\underline{s})\right)^{2} \\
& \times \theta\left((X(\underline{s})+\Delta A(\underline{s}))^{-1} \Delta A^{c}(\underline{s})\right) .
\end{aligned}
$$


Since $\Delta A^{c}(\underline{s}) \leqslant H^{-1}$, we may use (3.3) and (3.7) to bound the latter summation a.s. in absolute value by

$$
\begin{aligned}
\circ & \theta(0)^{\circ}\left(\max _{\underline{t}_{n-1} \leqslant s<t_{n}}(X(\underline{s})+\Delta A(\underline{s}))^{-1} \Delta A^{c}(\underline{s})\right) \\
& \times \int_{t_{n-1}}^{t_{n}}\left(x\left(s^{-}\right)+\Delta a(s)\right)^{-1} d a^{c}(s)=0 .
\end{aligned}
$$

Therefore by (3.7),

$$
\circ \sum_{1}=-\int_{t_{n-1}}^{t_{n}} x(s)^{-1} d a^{c}(s) \text { a.s. }
$$

Substitute (3.13) and (3.14) into (3.12) to see that

$$
\begin{aligned}
E\left(Z\left(\underline{t}_{n}\right) \mid L\left(\mathbb{Q}^{1} \times \mathbb{Q}_{\underline{t}_{n-1}}^{2}\right)\right)= & { }^{\circ} \bar{E}\left(Z\left(\underline{t}_{n}\right) \mid \mathbb{Q}^{1} \times \mathbb{Q}_{\underline{t}_{n-1}}^{2}\right) \text { a.s. } \\
= & Z\left(\underline{t}_{n-1}\right) \exp \left\{-\int_{t_{n-1}}^{t_{n}} x(s)^{-1} d a^{c}(s)\right\} \\
& \times \prod_{t_{n-1}<s \leqslant t_{n}}\left(1-\left(x\left(s^{-}\right)+\Delta a(s)\right)^{-1} \Delta a(s)\right) .
\end{aligned}
$$

Thus (3.10) follows immediately and the claim is proved. Using (3.10) inductively in (3.9) we obtain

$$
\begin{aligned}
P\left(\left(m\left(t_{0}\right), \ldots, m\left(t_{n}\right)\right) \in e_{0} B_{0} \times \cdots \times e_{n} B_{n}\right) & \\
=E(I((Z(0) x(0), & \left.\left.x\left(t_{1}\right), \ldots, x\left(t_{n}\right)\right) \in e_{0} B_{0} \times B_{1} \times \cdots \times B_{n}\right) \\
& \times 2^{-n} \prod_{i=1}^{n}\left(1+e_{i-1}^{\prime} e_{i}^{\prime} \exp \left\{-\int_{t_{i-1}}^{t_{i}} x(s)^{-1} d a^{c}(s)\right\}\right. \\
& \left.\left.\times \prod_{t_{i-1}<s \leqslant t_{i}}\left(1-\left(x\left(s^{-}\right)+\Delta a(s)\right)^{-1} \Delta a(s)\right)\right)\right),
\end{aligned}
$$

where $e_{i}^{\prime}=e_{i}$ if $i>0$ and $e_{0}^{\prime}=Z(0)$. Since $Z(0)$ is independent of $(a, x)$ and $P(Z(0)=1)=p=1-P(Z(0)=-1)$, (3.6) follows immediately. Finally $E(m(0) \mid a, x)=(2 p-1) x(0)$ a.s. because $m(0)=Z(0) x(0)$ (Lemma 3.4).

REMARK 3.12. Note that if $B_{1}, \ldots, B_{n}$ are any Borel subsets of $\mathbf{R}$, and $0 \leqslant t_{1}<$ $\cdots<t_{n}$, then $P\left(\left(m\left(t_{1}\right), \ldots, m\left(t_{n}\right)\right) \in B_{1} \times \cdots \times B_{n}\right)$ is a linear combination of the probabilities obtained in the above theorem.

It is now easy to prove Theorem 1.2. We give the proof in the local submartingale setting.

Proof of TheOREM 1.2. If $y(t) \geqslant 0$ is a local submartingale on $\left(X, \mathcal{G}, Q, \mathcal{G}_{t}\right)$ with Doob-Meyer decomposition $y=l+b$ ( $b$ is the increasing process), let $\left(\Omega^{1}, \mathbb{Q}^{1}, \bar{P}^{1}\right)$ $=\left({ }^{*} X,{ }^{*} \mathcal{G}, * \mathbb{Q}\right)$, and for $\underline{t}$ in $T=\left\{k \Delta t \mid k \in{ }^{*} \mathbf{N}_{0}\right\}$, let $\mathbb{Q}_{\underline{t}}^{1}=* \mathcal{G}_{\underline{t}}$. Let $\left(\Omega^{1}, \mathscr{F}^{1}, P^{1}, \mathscr{F}_{t}^{1}\right)$ be the adapted Loeb space generated by $\left(\Omega^{1}, \mathbb{Q}^{1}, \bar{P}^{1}, \mathbb{Q}_{t}^{1}\right)$. Let $x^{\overline{1}}=\operatorname{st}\left({ }^{*} y\right), n^{1}=\operatorname{st}\left({ }^{*} l\right)$ and $a^{1}=\operatorname{st}\left({ }^{*} b\right)$. Then by [H.P., Remark 10.10], $\left(x^{1}, a^{1}\right)$ is a well-defined $\mathscr{F}_{t}{ }^{1}$-adapted process and satisfies $\left(\left(x^{1}, a^{1}\right), \mathscr{F}_{\bullet}{ }^{l}\right) \equiv\left((y, b), \mathcal{G}_{.}\right)$. Moreover, the same result shows 
that $n^{1}$ is an ${\widetilde{F_{t}}}_{t}^{-}$-local martingale and, by Aldous [1, Theorem 19.6], $a^{1}$ is an increasing $\widetilde{r}_{t}^{1}$-predictable process. Hence $x^{1}(t)$ is a nonnegative local submartingale on $\left(\Omega^{1}, \widetilde{F}^{1}, P^{1}, \widetilde{F}_{t}^{1}\right)$ with Doob-Meyer decomposition $x^{1}=n^{1}+a^{1}$. Construct $x$ and $m$ on $\left(\Omega, \widetilde{T}, P,\left(\widetilde{T}_{t}\right)\right.$ as in $\S 2$. By Theorem $2.9 m$ is a local martingale and satisfies

$$
\left((|m|, a), \mathscr{F}_{\bullet}\right) \equiv\left(\left(x^{1}, a^{1}\right), \mathscr{\mathscr { F }}_{\bullet}^{1}\right) \equiv\left((y, b), \mathcal{G}_{\bullet}\right),
$$

where $a$ is the increasing process in the Doob-Meyer decomposition of $|m|$. Finally (1.2) and (1.3) follow from Theorem 3.11 by setting $p=\frac{1}{2}$ and noting that (3.15) implies that $(y, b)$ and $(x, a)$ have the same distribution.

4. An additional property of $m$. One could study several other properties of $m$, such as its jump structure across zero or its quadratic variation, by examining the corresponding internal properties of $M$. Instead of this we establish a key fact about $m$ (Corollary 4.4) which is shown in [12] to uniquely determine the probabilistic behaviour of the above properties when $m$ is any local martingale satisfying (1.1). In fact, in [12] we show that under an additional hypothesis on $x$ (which is readily checked and is satisfied in most cases of interest) any $m$ satisfying the conditions ((4.4) and (4.5)) of Corollary 4.4, as well as (1.1) and (1.2), must also satisfy (1.3) and hence is unique in law. Moreover in many cases of interest the conditions (4.4) and (4.5) are always satisfied (see $\S 4$ of [12]).

The notation is that introduced in $\S 2$.

Lemma 4.1. If $y(t, \omega)=y^{1}\left(t, \pi_{1}(\omega)\right)$ for some process $y^{1}$ on $\left(\Omega^{1}, \widetilde{F}^{1}, P^{1}\right)$ with sample paths in $D(\mathbf{R})$, then

$$
P(\exists \underline{t} \in \mathrm{ns}(T) \text { such that } \phi(\underline{t}+\Delta t)=-1,
$$

$$
\left.{ }^{\circ} X(\underline{t})>0,{ }^{\circ} \Delta A(\underline{t})=0 \text { and } \Delta y\left({ }^{\circ} \underline{t}\right) \neq 0\right)=0
$$

and

$$
\begin{aligned}
P\left(\exists t \in[0, \infty) \text { such that } m\left(t^{-}\right) m(t)<0,\right. & \\
\Delta a(t) & =0 \text { and } \Delta y(t) \neq 0)=0 .
\end{aligned}
$$

PRoOF. There is a sequence of $\widetilde{F}^{1}$-measurable random variables $\left\{U_{n}^{1}\right\}$ such that $\left\{t \mid \Delta y^{1}(t) \neq 0\right\} \subset\left\{U_{n}^{1}\right\}$ a.s. The probability in (4.1) is bounded above by

$$
\begin{aligned}
& P\left(\bigcup _ { n = 1 } ^ { \infty } \left\{\exists \underline{t} \in \mathrm{ns}(T) \text { such that } \underline{t} \approx U_{n}=U_{n}^{1} \circ \pi,\right.\right. \\
& \left.\left.\phi(\underline{t}+\Delta t)=-1,{ }^{\circ} X(\underline{t})>0,{ }^{\circ} \Delta A(\underline{t})=0\right\}\right),
\end{aligned}
$$

which is zero by Lemma 2.8. If $m\left(t^{-}\right) m(t)<0$ then clearly $\phi(t+\Delta t)=-1$ for some $\underline{t} \approx t$. Moreover (2.3) implies that ${ }^{\circ} X(t) \geqslant x\left(t^{-}\right) \wedge x(t)>0$ a.s. (the exceptional null set is independent of $t$ ) and hence (4.2) is an immediate consequence of (4.1). 
Recall that if $U$ is a stopping time with respect to a filtration $\left\{\hat{\mathscr{F}}_{t} \mid t \geqslant 0\right\}$, then $\hat{\mathscr{F}}_{U}$ is the $\sigma$-field generated by $\left\{A \cap\{t<U\} \mid A \in \hat{\mathscr{F}}_{t}, t \geqslant 0\right\} \cup \hat{\mathscr{F}}_{0}$.

Lemma 4.2. Let $\left(\Omega, \mathscr{F}, P, \hat{\mathscr{F}}_{t}\right)$ be the adapted Loeb space generated by $\left(\Omega, \mathcal{Q}, \bar{P}, \hat{\mathfrak{Q}}_{t}\right)$, where $\left\{\hat{\mathrm{Q}}_{\underline{t}}\right\}$ is some internal filtration.

(a) Then $U: \Omega \rightarrow[0, \infty)$ is an $\hat{\mathscr{F}}_{t}$-stopping time if and only if it has an $\hat{\mathscr{Q}}_{\underline{t}}$-lifting (see 2.2).

(b) If $V$ is an $\hat{\mathbb{Q}}_{t}$ lifting of $U$ and $U>0$ a.s., then $\hat{\mathscr{F}}_{U^{-}} \subset \sigma\left(\hat{\mathbb{Q}}_{V}\right) \vee \mathcal{N}$, where $\hat{\mathbb{Q}}_{V}=\left\{A \in \mathbb{Q} \mid A \cap\{V=\underline{t}\} \in \hat{\mathfrak{Q}}_{\underline{t}}\right.$ for all $\underline{t}$ in $\left.T\right\}$ and $\mathfrak{\Re}$ is the class of $P$-null sets.

Proof. The proof of (a) is in [H.P.] (Theorem 4.7(a)).

For (b), fix $t \geqslant 0$ and $A$ in $\hat{\mathscr{F}}_{t}$. By Lemma 2.1 there is a $t \approx t$ and $B \in \hat{\mathbb{Q}}_{\underline{t}}$ such that $P(A \Delta B)=0$. Since

$$
A \cap\{t<U\}=\bigcup_{m=1}^{\infty} B \cap\left\{\underline{t}+m^{-1}<V\right\} \quad \text { a.s., }
$$

and $B \cap\left\{\underline{t}+m^{-1}<V\right\} \in \hat{\mathbb{Q}}_{V}$, it follows that $A \cap\{t<U\} \in \sigma\left(\hat{\mathbb{Q}}_{V}\right) \vee \Re$. If $t=0$ we see that $\hat{\mathscr{F}}_{0} \subset \sigma\left(\hat{\mathscr{Q}}_{V}\right) \vee \Re$ and hence $\hat{\mathscr{F}}_{U^{-}} \subset \sigma\left(\hat{\mathscr{Q}}_{V}\right) \vee \Re$.

THEOREM 4.3. If

$$
P\left(\exists t \geqslant 0 \ni x\left(t^{-}\right)>0, x(t)=0 \text { and } \Delta a(t)>0\right)=0,
$$

then there is a sequence of disjoint predictable stopping times $\left\{T_{i}\right\}$ such that for each local martingale $y^{1}$ on $\left(\Omega^{1}, \mathscr{F}^{1}, P^{1}, \mathscr{F}_{t}^{1}\right)$ we have

$$
\left\{t \mid \Delta y(t) I\left(m\left(t^{-}\right) m(t)<0\right) \neq 0\right\} \subset\left\{T_{i}\right\} \text { a.s. }
$$

and

$$
E\left(\Delta y\left(T_{i}\right) I\left(m\left(T_{i}^{-}\right) m\left(T_{i}\right)<0, T_{i}<\infty\right) \mid \mathscr{F}_{T_{i}^{-}}\right)=0 \quad \text { a.s. }
$$

for all $i$ in $\mathbf{N}$, where $y(t, \omega)=y^{1}\left(t, \pi_{1} \omega\right)$.

Proof. Let $\left\{T_{i}^{1}\right\}$ be a sequence of disjoint $\left\{\mathscr{F}_{t}^{1}\right\}$-predictable stopping times such that $\left\{t \mid \Delta a^{1}(t) \neq 0\right\}=\left\{T_{i}^{1} \mid T_{i}^{1}<\infty, i \in \mathbf{N}\right\}$ a.s., and let $T_{i}=T_{i}^{1} \circ \pi_{i}$. Clearly $\left\{T_{i}\right\}$ is a sequence of $\left\{\mathscr{F}_{t}\right\}$-predictable times that satisfies (4.4) (by (4.2)). For $i$ fixed, let $U$ and $Y$ be $\mathbb{Q}^{1}$-measurable liftings of $T_{i}^{1}$ and $\Delta a^{1}\left(T_{i}^{1}\right) I\left(T_{i}^{1}<\infty\right)$, respectively, and define

$$
V_{n}^{1}=\min \left\{\underline{t} \geqslant U-n^{-1}|| \Delta A(\underline{t}-\Delta t)-Y \mid \leqslant n^{-1}\right\}
$$

$(\min \varnothing=+\infty)$. By saturation there is an $\mathbb{Q}^{1}$-measurable $V^{1}=V_{\gamma}^{1}: \Omega^{1} \rightarrow T \cup\{\infty\}$ $\left({ }^{\circ} \gamma=\infty\right)$ such that ${ }^{\circ} V^{1}=T_{i}^{1}$ a.s. and ${ }^{\circ} \Delta A^{1}\left(V^{1}-\Delta t\right)=\Delta a^{1}\left(T_{i}^{1}\right)$ a.s. on $\left\{T_{i}^{1}<\infty\right\}$. Let $V_{i}=V^{1} \circ \pi_{1}$ and $V_{i}^{\prime}=\left(V_{i}-\Delta t\right)^{+}$.

We claim that

$$
I\left(m\left(T_{i}^{-}\right) m\left(T_{i}\right)<0, T_{i}<\infty\right)=I\left(x\left(T_{i}^{-}\right)>0, \phi\left(V_{i}\right)=-1, T_{i}<\infty\right) \text { a.s. }
$$

If $m\left(T_{i}^{-}\right) m\left(T_{i}\right)<0$ and $T_{i}<\infty$ then w.p. $1, x\left(T_{i}^{-}\right)>0$ and $\phi(\underline{t})=-1$ for some $\underline{t} \approx T_{i}$. If $\underline{t} \neq V_{i}$ then w.p. $1,{ }^{\circ} \Delta A(\underline{t}-\Delta t)=0$ (recall $A$ is $S D J$ and ${ }^{\circ} \Delta A\left(V_{i}\right)=\Delta a\left(T_{i}\right)$ a.s. on $\left.\left\{T_{i}<\infty\right\}\right),{ }^{\circ} X(\underline{t}-\Delta t)>0$ (by (2.3) since $x\left(T_{i}^{-}\right) \wedge x\left(T_{i}\right)>0$ ) and $\phi(\underline{t})=$ -1 . By (4.2) this occurs with zero probability and hence $\phi\left(V_{i}\right)=-1$ (a.s.). Conversely if $x\left(T_{i}^{-}\right)>0, \phi\left(V_{i}\right)=-1$ and $T_{i}<\infty$, then by $(4.3) x\left(T_{i}\right)>0$ a.s. and 
therefore (2.3) implies that ${ }^{\circ} X(\underline{t})>0$ for all $\underline{t} \approx T_{i}$ a.s. Use (4.1) with $y=a$ to see that w.p. $1, \phi(\underline{t})=1$ for all $\underline{t} \approx T_{i}$ such that $\underline{t} \neq V_{i}$, and therefore for all $\underline{t}$ in $\left(V_{i}-\varepsilon\right.$, $\left.V_{i}+\varepsilon\right)-\left\{V_{i}\right\}$ for some real positive $\varepsilon(\omega)$. Therefore $m\left(T_{i}^{-}\right) m\left(T_{i}\right)<0$ a.s. (by the definition of $m$ ) and the claim is proven.

Let $\hat{\mathcal{Q}}_{\underline{t}}=\mathcal{Q}^{\prime} \times \hat{Q}_{\underline{t}}^{2}$ and note that $\left\{V_{i}^{\prime}=t\right\} \in \hat{\mathscr{Q}}_{0}$ and, by Lemma 4.2, $\sigma\left(\hat{\mathscr{Q}}_{V_{i}^{\prime}}\right) \vee \mathcal{X}$ $\supset \bar{F}_{T_{1}}$, where $\Re$ is the class of all $P$-null sets. Therefore by the above result and Lemma 2.1,

$$
\begin{aligned}
& E\left(\Delta y\left(T_{i}\right) I\left(m\left(T_{i}^{-}\right) m\left(T_{i}\right)<0, T_{i}<\infty\right) \mid \mathscr{F}_{T_{i}}\right) \\
& =E\left(\Delta y\left(T_{i}\right) I\left(x\left(T_{i}^{-}\right)>0, T_{i}<\infty\right)^{\circ} \bar{P}\left(\phi\left(V_{i}\right)=-1 \mid \hat{\mathscr{C}}_{V_{i}^{\prime}}\right) \mid \mathscr{F}_{T_{i}^{-}}\right) \text {a.s. }
\end{aligned}
$$

By considering separately the sets $\left\{V_{i}=\underline{s}\right\}$, it is clear that on $\left\{V_{i} \in T-\{0\}\right\}$,

$$
\bar{P}\left(\phi\left(V_{i}\right)=-1 \mid \hat{\mathcal{Q}}_{V_{i}^{\prime}}\right)=\Delta A\left(V_{i}-\Delta t\right)\left(2\left(X\left(V_{i}-\Delta t\right)+\Delta A\left(V_{i}-\Delta t\right)\right)\right)^{-1} .
$$

Thus we obtain

$$
\begin{aligned}
E\left(\Delta y\left(T_{i}\right)\right. & \left.I\left(m\left(T_{i}^{-}\right) m\left(T_{i}\right)<0, T_{i}<\infty\right) \mid \mathscr{F}_{T_{i}}\right) \\
= & E\left(\Delta y\left(T_{i}\right) I\left(x\left(T_{i}^{-}\right)>0, T_{i}<\infty\right) \Delta a\left(T_{i}\right)\right. \\
& \left.\times\left(2\left(x\left(T_{i}^{-}\right)+\Delta a\left(T_{i}\right)\right)\right)^{-1} \mid \mathcal{F}_{T_{i}}\right) \quad \text { a.s. } \\
& \quad\left(\text { since }{ }^{\circ} X\left(V_{i}-\Delta t\right)=x\left(T_{i}^{-}\right) \text {a.s. by }(2.3)\right) \\
= & I\left(x\left(T_{i}^{-}\right)>0, T_{i}<\infty\right) \Delta a\left(T_{i}\right)\left(2\left(x\left(T_{i}^{-}\right)+\Delta a\left(T_{i}\right)\right)\right)^{-1} E\left(\Delta y\left(T_{i}\right) \mid \mathscr{F}_{T_{i}^{-}}\right) \\
= & 0 \quad \text { a.s. }
\end{aligned}
$$

(the last because $T_{i}$ is predictable).

The following corollary is now immediate from the previous result and Lemma 2.5. Recall that $n(t)$ is the local martingale part of the Doob-Meyer decomposition of $x$.

Corollary 4.4. Assume (4.3) holds. If $\phi: D\left(\mathbf{R}^{2}\right) \rightarrow \mathbf{R}$ is bounded and measurable let $L_{\phi}(t)$ be a right continuous version of $E\left(\phi(x, a) \mid \mathscr{F}_{t}\right)$. Then there is a sequence of predictable stopping times $\left\{T_{i}\right\}$ with disjoint graphs such that (4.4) and (4.5) hold whenever $y \in\left\{L_{\phi} \mid \phi\right.$ bounded measurable $\} \cup\{n\}$.

5. Proof of Theorem 2.4. Our setting is that described at the beginning of $\S 2$ except that the superscript ${ }^{1}$ is dropped for notational convenience. The following lifting theorem is proved in [H.P.] (Theorem 5.6, Remark 5.7(c), and Remark 4.5(a)).

THEOREM 5.1. If $y(t)$ is a local martingale with respect to $\left\{\mathscr{F}_{t}\right\}$ and $y$ is reduced by $\left\{U_{n}\right\}$ (i.e. $y\left(U_{n} \wedge t\right)$ is a uniformly integrable martingale and $U_{n}$ increase to $\infty$ a.s.), then there is an internal filtration $\left\{\mathscr{G}_{\underline{t}}\right\}$, a sequence of $\mathscr{B}_{\underline{t}}$-stopping times $\left\{W_{n}\right\}$ and a ${ }_{\mathrm{S}} \underline{t}_{-}$local martingale lifting of $y, Y$, such that $\left\{W_{n}\right\}$ reduces $Y$ and ${ }^{\circ} W_{n}=U_{n}$ a.s.

(b) If $b(t)$ is a right-continuous $\widetilde{F}_{t}$-adapted process with nondecreasing sample paths, such that $b(0)=0$ a.s., then $b$ has $a \mathscr{\Re}_{\underline{\underline{t}}}^{-*}$-increasing lifting for every internal filtration $\left\{6_{\underline{1}}\right\}$. 
We will also use the fact that if $F: T \rightarrow{ }^{*} \mathbf{R}$ is an internal function then $F$ is $S D J$ if and only if $F$ is near-standard in the Skorokhod $J_{1}$ topology on $D(\mathbf{R})$, and the mapping st defined in $\$ 2$ is the standard part map on this class of functions (see [H.P., Theorem 2.6]).

Recall that $a$ is the increasing predictable process in the Doob-Meyer decomposition of the local submartingale $x$. The following lemma will be used to obtain a lifting of $a$ satisfying (2.2) and (2.3).

LEMMA 5.2. If $\left\{\mathscr{Q}_{t}\right\}$ is an internal filtration and $\left\{V_{n}\right\}$ is a sequence of $\mathscr{B}_{t}$-stopping times, then $a(t)$ has a $\mathscr{B}_{\underline{t}}^{-*}$-increasing lifting $A(\underline{t})$ such that $A(\underline{t}+\Delta t)$ is $\mathscr{B}_{\underline{\underline{t}}}^{\text {-adapted }}$ and ${ }^{\circ} A\left(V_{n}\right)=a\left({ }^{\circ} V_{n}\right)$ a.s. on $\left\{{ }^{\circ} V_{n}<\infty\right\}$ for all $n$.

Proof. Extend $\left\{V_{n} \mid n \in \mathbf{N}\right\}$ internally to ${ }^{*} \mathbf{N}$ and let $Y(\underline{t})=\sum_{n=1}^{\beta} 2^{-n} I\left(\underline{t} \geqslant V_{n}\right)$, where $\beta \in{ }^{*} \mathbf{N}-\mathbf{N}$. Let $a^{d}(t)=\sum_{s \leqslant t} \Delta a(s)$ and $a^{c}=a-a^{d}$. There is a disjoint sequence of positive predictable stopping times $\left\{T_{n}\right\}$ that covers the jumps of $a$, and for each $n$ there is a sequence of stopping times $\left\{T_{m}^{n} \mid m \in \mathbf{N}\right\}$ that announces $T_{n}$. If $V_{m}^{n}$ is a $\mathscr{B}_{\underline{t}}$-lifting of $T_{m}^{n}$, a routine saturation argument shows the existence of a $\mathscr{G}_{\underline{t}}$-stopping time $V_{\gamma(n)}^{n}\left(\gamma(n) \in{ }^{*} \mathbf{N}-\mathbf{N}\right)$ such that ${ }^{\circ} V_{\gamma(n)}^{n}=T_{n}$ a.s. and ${ }^{\circ} Y\left(V_{\gamma(n)}^{n}\right)=$ $\operatorname{st}(Y)\left(T_{n}^{-}\right)$a.s. Since $\Delta a\left(T_{n}\right)$ is $\mathcal{F}_{T_{n}^{-n}}$-measurable it has a nonnegative $\mathscr{B}_{V_{\gamma(n)}^{n}}$-measurable lifting $A^{n}$ by Lemma 4.2. Let

$$
A_{m}(\underline{t})=\sum_{n=1}^{m} A^{n} I\left(\underline{t}>V_{\gamma(n)}^{n}\right) \quad \text { and } \quad a_{m}(t)=\sum_{n=1}^{m} \Delta a\left(T_{n}\right) I\left(t \geqslant T_{n}\right) .
$$

If $A^{d}$ is a $\mathscr{B}_{t^{-}}$-increasing lifting of $a^{d}$ (see Theorem 5.1) and $\rho$ denotes a metric inducing the Skorokhod $J_{1}$ topology on $D(\mathbf{R})$, then, since $\operatorname{st}\left(A_{m}\right)=a_{m}$ a.s., Theorem 2.6 in [H.P.] implies that

$$
\lim _{m \rightarrow \infty}{ }^{\circ} \rho\left(A_{m}, A^{d}\right)=\lim _{m \rightarrow \infty} \rho\left(a_{m}, a^{d}\right)=0 \text { a.s. }
$$

Extend $\left\{\left(A_{m}, V_{\gamma(m)}^{m}, A^{m}\right) \mid m \in \mathbf{N}\right\}$ internally to ${ }^{*} \mathbf{N}$ and select $\alpha$ in ${ }^{*} \mathbf{N}-\mathbf{N}$ such that $A_{\alpha}(t+\Delta t)$ is $\mathscr{B}_{\underline{t}}$-adapted, $A_{\alpha}(\underline{t}, \omega)$ is nondecreasing on $T$ and is zero at $\underline{t}=0$ for all $\omega, \Delta A_{\alpha}\left(V_{\gamma(m)}^{m}\right)=A^{m}$ for $m \leqslant \alpha$, and ${ }^{\circ} \rho\left(A_{\alpha}, A^{d}\right)=0$. The last condition and Theorem 2.6 in [H.P.] imply that $A_{\alpha}$ is an $S D J$ lifting of $a^{d}$. If $A^{c}$ is a $\mathscr{B}_{\underline{t}}$ - $^{*}$-increasing lifting of $a^{c}$ then the continuity of $a^{c}$ implies that ${ }^{\circ} A^{c}(\underline{t})=a^{c}\left({ }^{\circ} \underline{t}\right)$ for all $\underline{t}$ in $\operatorname{ns}(T)$ a.s. Therefore $A(\underline{t})=A^{c}\left((\underline{t}-\Delta t)^{+}\right)+A_{\alpha}(\underline{t})$ is a $\Re_{\underline{t}^{-}}{ }^{-}$-increasing lifting of $a$ and $A(\underline{t}+\Delta t)$ is clearly $\Re_{\underline{t}}$-adapted. Suppose ${ }^{\circ} A\left(V_{m}\right) \neq a\left({ }^{\circ} V_{m}\right)$ and ${ }^{\circ} V_{m}<\infty$. Then w.p. 1, $\Delta a\left({ }^{\circ} V_{m}\right)>0$ and therefore ${ }^{\circ} V_{m}=T_{n}$ for some $n$. Since $A$ is $S D J$ and ${ }^{\circ} \Delta A\left(V_{\gamma(n)}^{n}\right)$ $={ }^{\circ} A^{n}=\Delta a\left(T_{n}\right)>0$ a.s., it follows that $V_{m} \leqslant V_{\gamma(n)}^{n}$ a.s. This implies that ${ }^{\circ} Y\left(V_{\gamma(n)}^{n}\right)$ $\geqslant{ }^{\circ} Y\left(V_{m}\right) \geqslant \operatorname{st}(Y)\left(T_{n}^{-}\right)+2^{-m}$. Recalling that ${ }^{\circ} Y\left(V_{\gamma(n)}^{n}\right)=\operatorname{st}(Y)\left(T_{n}^{-}\right)$a.s., we see that our assumption can only hold on a null set, that is ${ }^{\circ} A\left(V_{m}\right)=a\left({ }^{\circ} V_{m}\right)$ a.s. on $\left\{{ }^{\circ} V_{m}<\infty\right\}$ for all $m$, as required.

LEMMA 5.3. If $\left\{9_{\underline{t}}\right\}$ is an internal filtration and $A$ is $a B_{t_{t}}{ }^{*}$-increasing lifting of $a$ and $\left\{V_{m} \mid m \in \mathbf{N}\right\}$ is a sequence of $\mathscr{B}_{\underline{t}}$-stopping times such that $\lim _{m \rightarrow \infty}{ }^{\circ} V_{m}=\infty$ a.s., $E\left(a\left({ }^{\circ} V_{m}\right)\right)<\infty$ and ${ }^{\circ} A\left(V_{m}\right)=a\left({ }^{\circ} V_{m}\right)$ a.s., then for some $\gamma$ in ${ }^{*} \mathbf{N}-\mathbf{N},(A \wedge \gamma)\left(V_{m}\right)$ is $S$-integrable for all $m$ in $\mathbf{N}$. 
Proof. If $Y_{m}$ is an $S$-integrable lifting of $a\left({ }^{\circ} V_{m}\right)$, then for all $n$ in $\mathbf{N}$,

$$
\bar{E}\left(\max _{m \leqslant n}\left|A\left(V_{m}\right) \wedge n-Y_{m} \wedge n\right|\right)<2^{-n} \text {. }
$$

Therefore (5.1) holds when $n$ is replaced by some $\gamma$ in $* \mathbf{N}-\mathbf{N}$ and the result follows.

Proof of Theorem 2.4. Let $\left\{U_{n}\right\}$ be a sequence of finite stopping times increasing to $\infty$ a.s. such that $E\left(a\left(U_{n}\right)\right)<\infty$ and $n\left(U_{n} \wedge t\right)$ is a uniformly integrable martingale. By Theorem 5.1 there is an internal filtration $\left\{\mathscr{B}_{t}\right\}$, a sequence of $\mathscr{B}_{t}$-stopping times $\left\{W_{n}\right\}$, and a $\mathscr{T}_{\underline{t}}$-local martingale lifting of $n, N_{1}$, such that $\left\{W_{n}\right\}$ reduces $N_{1}$ and ${ }^{\circ} W_{n}=U_{n}$ a.s. Let $\left\{W_{n}^{\prime}\right\}$ be a sequence of $\Re_{t}$-stopping times such that $\left\{\left.\underline{t}\right|^{\circ}\left|\Delta N_{1}(\underline{t}-\Delta t)\right|>0\right\} \subset\left\{W_{n}^{\prime}\right\}$ a.s. and let $A_{1}$ be the $\mathscr{B}_{t^{-}}$-increasing lifting of $a$ obtained in Lemma 5.2 with $\left\{V_{n}\right\}=\left\{W_{n}\right\} \cup\left\{W_{n}^{\prime}\right\}$. Since for a.a. $\omega,{ }^{\circ} W_{n}^{\prime}<\infty$ implies ${ }^{\circ} A_{1}\left(W_{n}^{\prime}\right)=a\left({ }^{\circ} W_{n}^{\prime}\right)$, clearly $A_{1}$ and $N_{1}$ satisfy (2.3). Note that $E\left(a\left({ }^{\circ} W_{n}\right)\right)=$ $E\left(a\left(U_{n}\right)\right)<\infty$. Therefore Lemma 5.3 allows us to assume that $\left\{W_{n}\right\}$ reduces $A_{1}$ and that $\sup _{(\underline{\underline{t}}, \omega)} A_{1}(\underline{t}, \omega) \in{ }^{*} \mathbf{R}$, by replacing $A_{1}$ with $A_{1} \wedge \gamma$ for some $\gamma$ in $* \mathbf{N}-\mathbf{N}$. If $X_{1}=N_{1}+A_{1}$, then $X_{1}$ is an $S D$ lifting of $x$ and since $N_{1}$ and $A_{1}$ satisfy (2.3), ${ }^{\circ} X_{1}(\underline{t}) \geqslant 0$ for all $\underline{t}$ in $\mathrm{ns}(T)$ a.s. Choose a positive infinitesimal $\delta$ such that $X_{1}(\underline{t})+\delta \geqslant 0$ for all $\underline{t}$ in $\mathrm{ns}(T)$ a.s. and let $X(\underline{t})=\left(X_{1}(\underline{t})+\delta\right)^{+}+\delta$. Then

$$
\begin{aligned}
\Delta X(\underline{t})= & I\left(X_{1}(\underline{t})+\delta \geqslant 0\right)\left(\Delta X_{1}(\underline{t})+\left(X_{1}(\underline{t}+\Delta t)+\delta\right)^{-}\right) \\
& +I\left(X_{1}(\underline{t})+\delta<0\right)\left(X_{1}(\underline{t}+\Delta t)+\delta\right)^{+},
\end{aligned}
$$

and therefore,

$$
\begin{aligned}
X(\underline{t})= & (X(0)+\delta)^{+}+\delta+\left(N_{1}(\underline{t})-N_{1}(0)\right)+N_{2}(\underline{t}) \\
& +\int I\left(\underline{s}<\underline{t}, X_{1}(\underline{s})+\delta \geqslant 0\right) d \nu_{A_{1}}+A_{2}(\underline{t})
\end{aligned}
$$

where

$$
\begin{aligned}
A_{2}(\underline{t})= & \sum_{\underline{s}<\underline{t}} I\left(X_{1}(\underline{s})+\delta \geqslant 0\right)\left(X_{1}(\underline{s}+\Delta t)+\delta\right) \\
& +I\left(X_{1}(\underline{s})+\delta<0\right)\left(X_{1}(\underline{s}+\Delta t)+\delta\right)^{+}
\end{aligned}
$$

and

$$
N_{2}(\underline{t})=-\sum_{\underline{s}<\underline{t}} I\left(X_{1}(\underline{s})+\delta<0\right) \Delta N_{1}(\underline{s}) .
$$

The choice of $\delta$ implies $A_{2}(\underline{t})=N_{2}(\underline{t})=0$ for all $\underline{t}$ in $\operatorname{ns}(T)$ a.s. By (5.2) $\left\{W_{n}\right\}$ reduces $N_{2}+A_{2}$ since it reduces the other terms in (5.2). Since $N_{2}$ is an internal martingale, and since we may assume $W_{n} \leqslant \gamma$ for some $\gamma$ in ${ }^{*} \mathbf{N}-\mathbf{N}$ (replace $W_{n}$ by $\left.W_{n} \wedge \gamma\right)$, it follows that

$$
\begin{aligned}
{ }^{\circ} \bar{E}\left(\left|N_{2}\left(W_{n}\right)\right|\right) & =2 \circ \bar{E}\left(N_{2}^{+}\left(W_{n}\right)\right) \leqslant 2 \circ \bar{E}\left(\left|N_{2}\left(W_{n}\right)+A_{2}\left(W_{n}\right)\right|\right) \\
& =2 E\left({ }^{\circ}\left|N_{2}\left(W_{n}\right)+A_{2}\left(W_{n}\right)\right|\right) \quad\left(\left\{W_{n}\right\} \text { reduces } N_{2}+A_{2}\right) \\
& =0 .
\end{aligned}
$$

Therefore $\left\{W_{n}\right\}$ reduces $N_{2}$ and hence also $A_{2}$. If $A_{2}^{p}(\underline{t})=\sum_{\underline{s}<\underline{t}} \bar{E}\left(\Delta A_{2}(\underline{s}) \mid \mathscr{G}_{\underline{s}}\right)$, then $\bar{E}\left(A_{2}^{p}\left(W_{n}\right)\right)=\bar{E}\left(A_{2}\left(W_{n}\right)\right) \approx 0$. Hence, $A_{2}^{p}(\underline{t}) \approx 0$ for all $\underline{t}$ in $\operatorname{ns}(T)$ a.s. and $A_{2}^{p}$ is 
reduced by $\left\{W_{n}\right\}$. Define

$$
N(\underline{t})=\left(X_{1}(0)+\delta\right)^{+}+\delta+N_{1}(\underline{t})-N_{1}(0)+N_{2}(\underline{t})+A_{2}(\underline{t})-A_{2}^{p}(\underline{t})
$$

and

$$
A(\underline{t})=\int I\left(\underline{s}<t, X_{1}(\underline{s})+\delta \geqslant 0\right) d \nu_{A_{1}}+A_{2}^{p}(\underline{t}) .
$$

Then $\left\{W_{n}\right\}$ reduces $N$ and $A, A(\underline{t}+\Delta t)$ is $\Re_{\underline{t}}$-adapted, and for a.a. $\omega, N(\underline{t}) \approx N_{1}(\underline{t})$ and $A(\underline{t}) \approx A_{1}(\underline{t})$ for all $\underline{t}$ in $\mathrm{ns}(T)$. It follows that $\left(X, A, N,\left\{W_{n}\right\}\right)$ satisfies the required conditions. (Note that $X$ satisfies (2.4) since $\sup _{(t, \omega)}\left|X_{1}(\underline{t}, \omega)\right| \in{ }^{*} \mathbf{R}$.)

ADDED IN PROOF. Recently Hoover and Keisler [15] have shown that if $x$ is a (local) submartingale defined on an adapted Loeb space $\left(\Omega, \mathscr{F}, P, \mathscr{F}_{t}\right)$, rich enough to support an $\mathscr{F}_{t}$-Brownian motion then there is a (local) martingale, $m$, such that $|m|=x$ a.s. That is, there is no need to enlarge such a Loeb space. They use our results in their proof.

\section{REFERENCES}

1. D. J. Aldous, A concept of weak convergence for stochastic processes viewed in the Strasbourg manner (preprint).

2. R. M. Anderson, A nonstandard representation for Brownian motion and Itô integration, Israel J. Math. 25 (1976), 15-46.

3. M. T. Barlow, Construction of a martingale with given absolute value, Ann. Probab. 9 (1981), 314-320.

4. M. T. Barlow and M. Yor, Sur la construction d'une martingale continue, de valeur absolue donnée, Sém. Probabilités XIV, Lecture Notes in Math., vol. 784, Springer-Verlag, Berlin, 1980.

5. P. Billingsley, Convergence of probability measures, Wiley, New York, 1968.

6. D. Gilat, Every nonnegative submartingale is the absolute value of a martingale, Ann. Probab. 5 (1977), 475-481.

7. D. Hoover and E. Perkins, Nonstandard construction of the stochastic integral and applications to stochastic differential equations. I, II, Trans. Amer. Math. Soc. (to appear).

8. H. J. Keisler, An infinitesimal approach to stochastic analysis, Mem. Amer. Math. Soc. (to appear).

9. P. A. Loeb, Conversion from nonstandard to standard measure spaces and applications in probability theory, Trans. Amer. Math. Soc. 211 (1975), 113-122.

10. , An introduction to nonstandard analysis and hyperfinite probability theory, Probabilistic Analysis and Related Topics (A. Barucha-Reid, editor), Academic Press, New York, 1979.

11. B. Maisonneuve, Martingales de value absolue donnée, d'apres Protter and Sharpe, Sém. Probabilités XIII, Lecture Notes in Math., vol. 721, Springer-Verlag, Berlin, 1979.

12. E. Perkins, Uniqueness of a martingale with a given absolute value, Z. Wahrsch. Verw. Gebiete 56 (1981), 255-281.

13. P. Protter and M. J. Sharpe, Martingales with given absolute value, Ann. Probab. 7 (1979), 1056-1958.

14. C. J. Stone, Weak convergence of stochastic processes defined on semi-infinite time intervals, Proc. Amer. Math. Soc. 14 (1963), 694-696.

15. D. Hoover and H. J. Keisler, Adapted probability distributions (preprint).

Department of Mathematics, University of British Columbia, Vancouver, British Columbia, CANADA V6T 1 Y4 